\title{
El Automóvil y la Arquitectura de sus Infraestructuras en la ciudad de Albacete
}

José Luis Fuentes-Bargues ${ }^{1}$

Recibido: 17-11-2020 | en su versión final: 08-03-2021

Resumen

El desarrollo y la expansión del automóvil a principios de siglo XX originó la aparición de nuevas infraestructuras tales como las estaciones de servicio, los garajes, los lavaderos, etc. Estos espacios, con sus programas de necesidades específicos, fueron un reflejo de la modernidad y del poder adquisitivo de la nueva burguesía de las grandes ciudades, y fueron diseñados por los grandes arquitectos del eclecticismo y modernismo de la época. El objetivo del presente trabajo es el análisis y descripción del desarrollo de los principales espacios relacionados con el automóvil durante principios del siglo XX en la ciudad de Albacete, mostrando sus características funcionales, su distribución y su arquitectura. Para el desarrollo del trabajo se ha analizado el marco urbanístico, social y económico de la época a través de monografías y datos procedentes del Archivo Histórico Provincial de Albacete y para el análisis de las infraestructuras se han analizado los expedientes de licencia de construcción de edificios e infraestructuras del Archivo Histórico Municipal de Albacete. Este estudio permite conocer bajo el prisma del automóvil la historia de las infraestructuras del primer tercio del siglo XX en la ciudad de Albacete. A diferencia de lo que sucedió en grandes ciudades como Madrid, Barcelona o Valencia, la introducción y desarrollo del automóvil en la ciudad de Albacete fue más lento, y las características de la ciudad influyeron en un desarrollo de garajes individuales y de pequeño tamaño, destinados principalmente a guarda y custodia de los vehículos.

Palabras clave: Vehículos; garajes; modernismo; eclecticismo

Citación

Fuentes-Bargues, J.L. (2021). El Automóvil y la Arquitectura de sus Infraestructuras en la ciudad de Albacete. ACE: Architecture, City and Environment, 16(46), 9878. DOl: http://dx.doi.org/10.5821/ace.16.46.9878

\section{The Automobile and the Architecture of its Infrastructure in the City of Albacete}

Abstract The development and expansion of the automobile at the beginning of the 20th century gave rise to the appearance of new infrastructures such as service stations, garages, car washes, etc. These spaces, with their specific needs programs, were a reflection of modernity and the purchasing power of the new bourgeoisie in the big cities, and were designed by the great architects of eclecticism and modernism of the time. The aim of this work is to analyze and describe the development of the main spaces related to the automobile during the early 20th century in the city of Albacete, showing its functional characteristics, its distribution and its architecture. For the development of the work, the urban, social and economic framework of the time has been analyzed through monographs and data from the Albacete Provincial Historical Archive. For the analysis of the infrastructures, the files for the license to build buildings and infrastructures of the Albacete Municipal Historical Archive have been analyzed. This study allows to know under the prism of the automobile the history of the infrastructures of the first third of the 20th century in the city of Albacete. Unlike what happened in large cities like Madrid, Barcelona or Valencia, the introduction and development of the automobile in the city of Albacete was slower, and the characteristics of the city influenced the development of individual and small garages, mainly for the care and custody of vehicles.

Keywords: Cars; garages; modernism; eclecticism

${ }^{1}$ Dr. Ingeniero Industrial y Arquitecto Técnico, PRINS Research Center, Universitat Politècnica de València (ORCiD: 0000-0003-4877-3291, Scopus Author ID: 56030453700, Researcher ID: ABG-25412020). Correo de contacto: jofuebar@dpi.upv.es 


\section{Introducción}

La velocidad de desarrollo y aplicación de nuevas tecnologías en la sociedad actual se ha incrementado exponencialmente, especialmente en el siglo XXI con la aparición de las denominadas "nuevas tecnologías", lo que ha supuesto el abandono y desuso de tecnologías industriales utilizadas hasta hace muy poco tiempo.

Esta evolución de la sociedad ha desarrollado progresivamente desde finales del siglo XX un interés cultural creciente por comprender algunos de los eslabones de la cadena que nos han conducido a los niveles de calidad de vida actual (Muñoz-Carrión, 2012; Elinbaum, 2018) y se ha desarrollado un mayor interés por la conservación y salvaguarda del patrimonio cultural industrial, que se ha traducido tanto en políticas gubernamentales (en España el Plan Nacional de Patrimonio Industrial elaborado por el Ministerio de Educación, Cultura y Deporte) como en el aumento de investigaciones sobre patrimonio industrial e historia de la tecnología en el mundo académico (Aguilar Civera, 2003; Gutiérrez-Mozo et al., 2019). Con esta motivación cultural y académica se plantea una investigación sobre la arquitectura y las infraestructuras relacionadas con el desarrollo y expansión del automóvil a principios del siglo XX.

El automóvil llegó con mayor celeridad a ciudades como Madrid, Barcelona o Valencia, generando una serie de nuevas infraestructuras tales como estaciones de servicio, garajes, lavaderos, etc., que pronto se introdujeron en el día a día de la sociedad de inicio del siglo XX y que todavía perduran hasta la actualidad. A parte de la paulatina pavimentación de calles en las ciudades y de carreteras, la principal infraestructura que se desarrolló fueron los garajes, que servían como lugares de guarda y custodia, pero también era un reflejo de prosperidad y modernidad para la burguesía de las ciudades (Fuentes-Bargues, 2014; Martínez-Verón, 2017).

No hay mucha bibliografía académica respecto a estas infraestructuras, Mac Donald (2007) realizó un análisis sobre la evolución de los garajes durante el siglo XX en Estados Unidos, Fuentes-Bargues analizó en 2014 la arquitectura de los garajes en la ciudad de Valencia en el primer tercio del siglo XX, Muñoz (2018) realizó un estudio sobre la incidencia del automóvil en la arquitectura comercial de la ciudad de Buenos Aires, y algunas reseñas se pueden encontrar en los trabajos de Benito Goerlich (1992) en su estudio de la arquitectura ecléctica y modernista en Valencia y de Martínez Verón (2017), donde plasma alguna de las instalaciones en su blog sobre la Arquitectura del siglo XX en Zaragoza.

Estos trabajos describen con mayor o menor profundidad cómo se desarrollaron las infraestructuras del automóvil en grandes ciudades, pero no existen trabajos que describan y analicen la influencia del automóvil en el desarrollo de sus infraestructuras en ciudades más pequeñas cómo es el caso de Albacete.

El presente trabajo tiene como objetivo analizar y describir el desarrollo de los principales espacios relacionados con el automóvil durante principios del siglo XX en la ciudad de Albacete, describiendo su funcionalidad, su distribución y su arquitectura. El voraz desarrollo urbanístico y edificatorio de Albacete durante los años sesenta y setenta supuso la demolición de muchas edificaciones de finales del siglo XIX y principios del siglo XX, por lo que este estudio supone una aportación al conocimiento y a la historia de la arquitectura y de la ciudad de Albacete.

La metodología seguida en el presente estudio se ha dividido en cuatro fases. La primera fase consistió en el análisis del estado del arte, es decir una búsqueda en los repositorios científicos de los trabajos relacionados con el automóvil y sus infraestructuras, así como de trabajos relacionados con el marco social, económico, urbanístico y arquitectónico de la ciudad de Albacete en el inicio 
del siglo XX. La segunda fase del estudio consistió en la búsqueda de expedientes de obras en el Archivo Histórico Municipal de Albacete (en adelante, AMA) en el período comprendido entre 1900 y 1930. Se selecciona como período de estudio hasta el año 1930 porque en esa fecha el automóvil es un elemento totalmente consolidado, tanto en Albacete como en España, y porque es el mismo horizonte temporal que otros trabajos han tomado para su análisis (Fuentes-Bargues, 2014). La tercera fase consistió en un trabajo de campo en la ciudad, comprobando la existencia de alguna de las obras identificadas, y la cuarta fase del trabajo consistió en la elaboración de este artículo.

\section{La ciudad de Albacete en el siglo XX}

La ciudad de Albacete está situada en el sudeste de la península ibérica, y por su situación ha sido paso obligatorio entre las rutas entre el centro peninsular y la zona Mediterránea, así como de las rutas entre el Mediterráneo y Andalucía. Esta posición intermedia en las rutas le hado dado históricamente un carácter a la ciudad de "parada y fonda" (Gutiérrez-Mozo, 2001), influyendo en la estructura urbana de la ciudad claramente lineal hasta la llegada del ferrocarril en el año 1855. La llegada de la línea férrea supuso una barrera para el crecimiento por el Norte de la ciudad, que condicionó el urbanismo posterior de la ciudad.

La ciudad de Albacete está ubicada en una llanura (Al Basit, significa el Llano) donde se elevan tres pequeños promontorios que han permitido históricamente una doble función: la defensiva y la higiénica (Gutiérrez-Mozo, 2012). Estos promontorios son el Alto de la Villa o Villacerrada, el Alto de las Carretas y el Cerro de San Juan, y en la actualidad conforman el centro histórico de la ciudad. Durante el siglo XIX, junto a la citada llegada del ferrocarril a la ciudad, la desamortización eclesiástica y civil supusieron la apertura de calles y la fragmentación de grandes manzanas ocupadas por edificios eclesiásticos en el este de la ciudad, permitiendo así disponer de un entramado viario más moderno y de grandes bolsas de suelo para la construcción de viviendas (Panadero Moya, 1984). Esta nueva situación se plasmó en el Plan de Alineación General de las calles de la ciudad de Albacete, 1882-1886, realizado por el arquitecto D. Juan Antonio Peryronnet Rodríguez.

A estos cambios urbanísticos se le unieron las grandes infraestructuras municipales necesarias para la concepción de una ciudad moderna (Figura 1). El 1 de abril de 1888 llegó la electricidad a Albacete, sustituyendo así el alumbrado de farolas de aceite, en el año 1905 se inauguró la red de abastecimiento de agua potable y el año 1909 la red de alcantarillado, diseñada por el arquitecto municipal D. Francisco Manuel Martínez Villena.

Este cambio en la ciudad generó un aumento de la actividad económica y la consecuente necesidad de mano de obra, tanto para el sector terciario, como para las actividades industriales, muy relacionadas con el sector primario y con los bienes de consumo (Romero Soriano et al., 1980). Durante el primer tercio del siglo XX se produjo en la ciudad de Albacete el mayor crecimiento demográfico de su historia, pasando de 21.512 habitantes en el año 1900 a 41.885 habitantes en el año 1930 (INE, 1955).

Este aumento de población generó una necesidad de vivienda y el consecuente aumento de la actividad constructiva, y la necesidad de un crecimiento regulado y acorde a las exigencias del nuevo siglo. En el año 1904 se promulgaron unas Ordenanzas Municipales, cuyo Título 10 regulaba en catorce capítulos todo lo referente a urbanismo (Gutiérrez Mozo, 2004). En el año 1908 el arquitecto municipal D. Francisco Manuel Martínez Villena redactó el Plan de Alineaciones. Este Plan planteaba dos ensanches de la población, uno hacia el Norte, hasta la vía del ferrocarril y otro hacia el Sur, ocupando la zona baja de huertas y la zona del actual parque Abelardo Sánchez. El parque 


\section{ACE Architecture, City and Environment}

es uno de los emblemas de la actual ciudad, fue construido en 1911 y bautizado como Parque de Canalejas, siendo alcalde D. Abelardo Sánchez y en él se plantaron más de 12.000 pinos y 400 árboles de distintas especies, y desde su concepción y construcción condicionó la trama urbanística de la ciudad (Figura 2).

Figura 1. Plano de la ciudad de Albacete en 1905

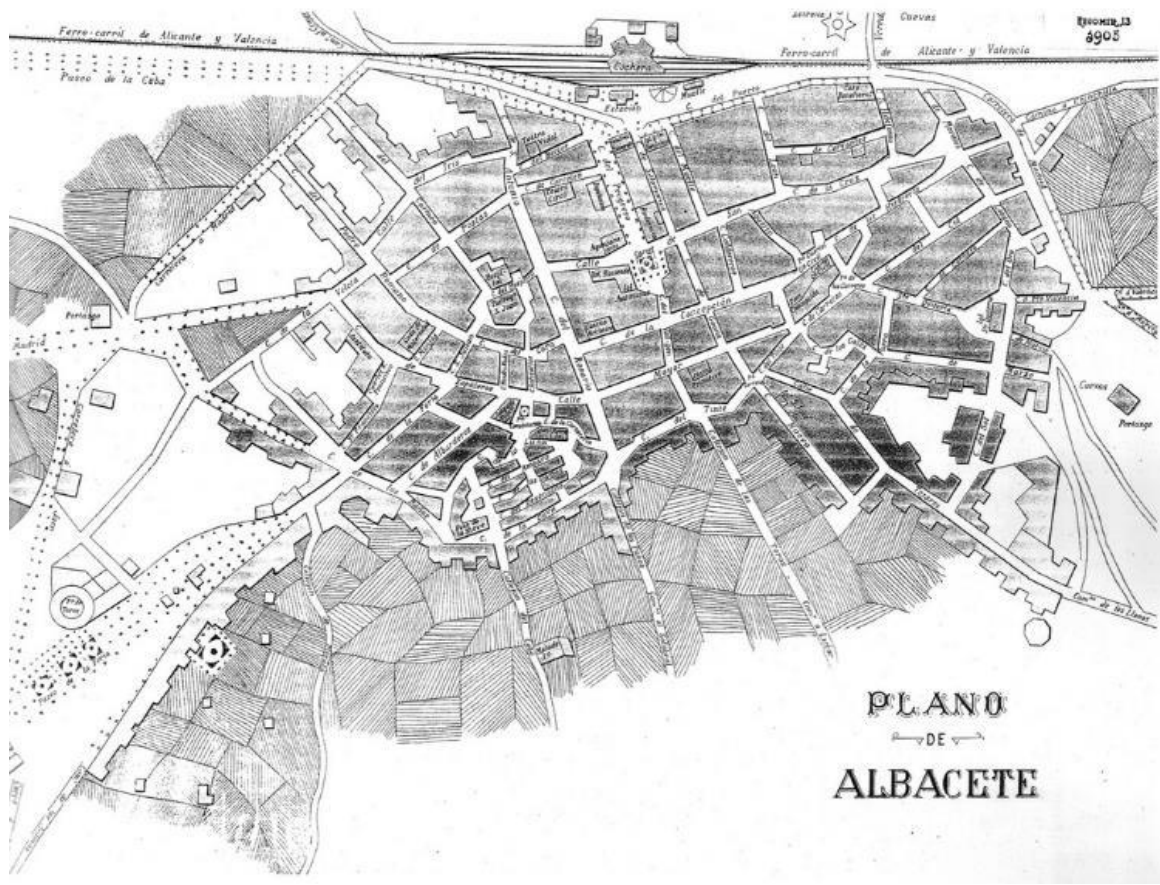

Fuente: Archivo Histórico Provincial de Albacete (AHPA).

Figura 2. Plano de Albacete de 1915

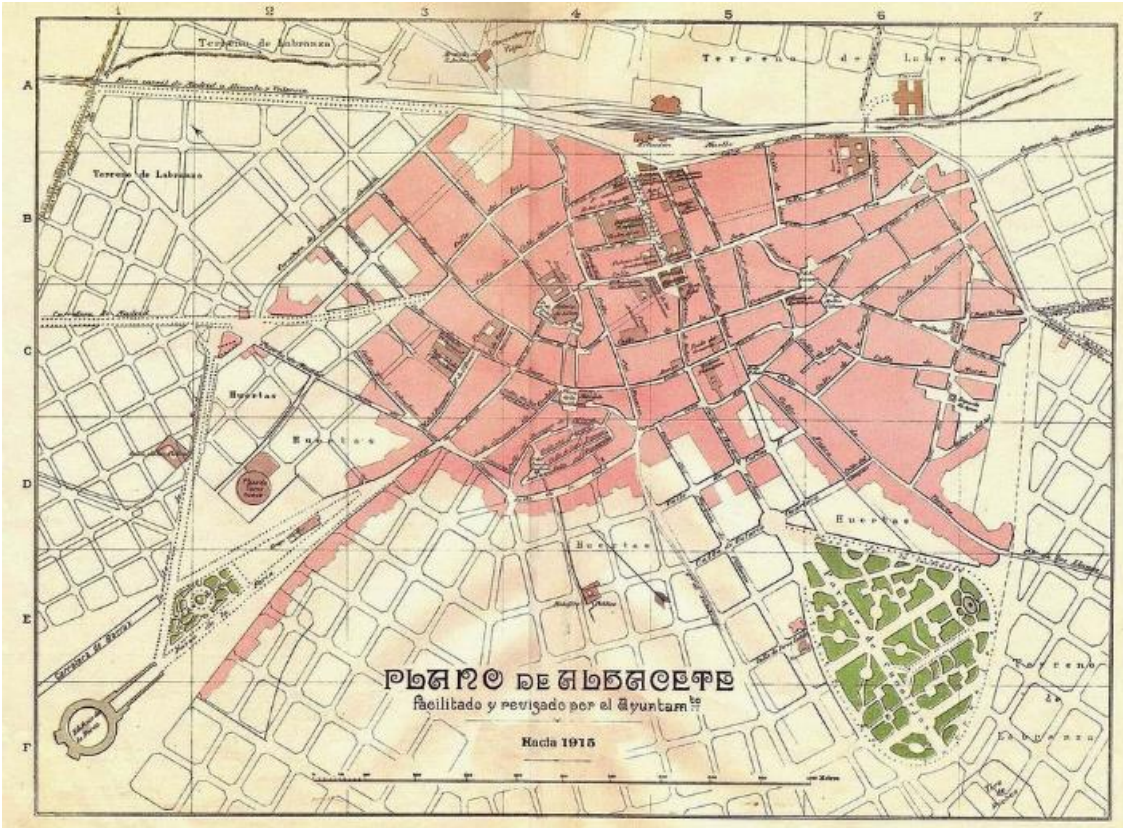

Fuente: AHPA. 
En el año 1922, los arquitectos D. Julio Carrilero Prats y D. Manuel Muñoz Cayasús redactaron el primer Plan de Ensanche de Albacete. Este Plan rodea de una amplia retícula la ciudad consolidada, el Albacete antiguo (Villacerrada, el Alto de las Carretas y el Cerro de San Juan y la Feria) y el Albacete desarrollado con el Plan de Alineaciones (barrio de la Industria y entorno del Parque) (Gutiérrez Mozo, 2004). El Plan propone una malla rectangular con un tamaño de manzana de $70 \times$ $80 \mathrm{~m}$, calles de 10, 12 y $14 \mathrm{~m}$, sectorizando la ciudad en ocho zonas, delimitadas por las carreteras y caminos que confluyen en la ciudad de Albacete.

En 1929, el Plan de Ensanche sufre otra modificación (Figura 3), redactada por el arquitecto D. Miguel Ortiz e Iribas, que afecta principalmente a las mallas entre el Paseo de la Feria y la calle del Rosario, y desarrolla una infraestructura urbana característica de la ciudad de Albacete, la Circunvalación, que enlaza por el Sur la carretera de Madrid con las de Murcia y Alicante.

Figura 3. Plano de Albacete con la trama del Ensanche de 1929 editado por los Talleres Gráficos La Minerva

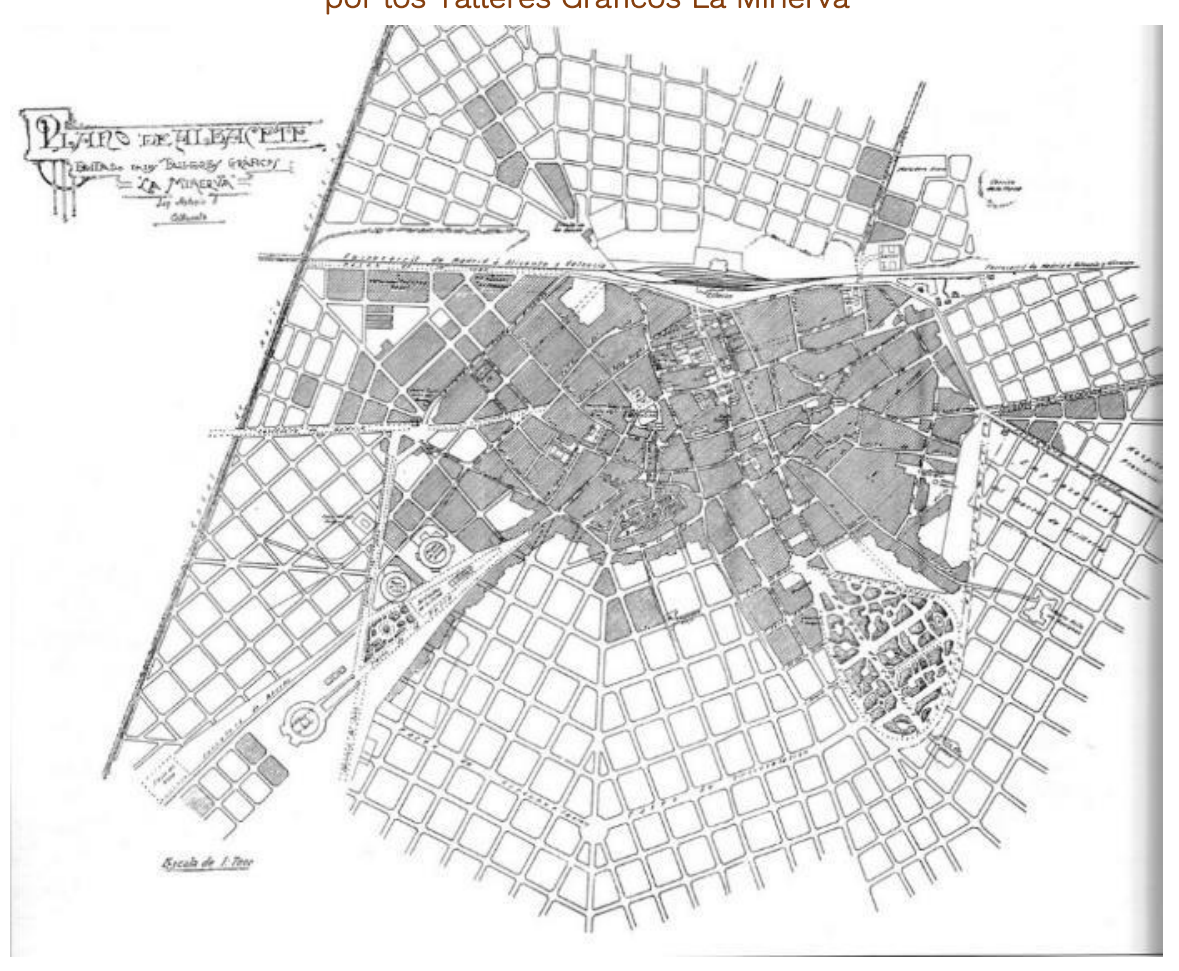

Fuente: AHPA

El crecimiento demográfico, económico y urbano de la ciudad, junto con una burguesía con un alto poder económico y político-institucional, generaron un elevado desarrollo arquitectónico de la ciudad, pasando de 3.691 edificaciones en el año 1900 a 5.856 edificaciones en el año 1930 (Romero Soriano et al., 1980).

Este auge arquitectónico supuso la ejecución de numerosas obras de carácter ecléctico y modernista, principalmente en el centro de la ciudad, si bien la transformación de la ciudad durante las décadas de los años sesenta y setenta del siglo XX hicieron que no todas ellas se conserven en la actualidad (Romero Soriano et al., 1980; Magán Perales, 1997). Entre las principales joyas arquitectónicas del período de estudio que se conservan en la ciudad se encuentran el Quiosco de la Feria (1912), el Museo de la Cuchillería (1912) y el Gran Hotel (1915), todas del arquitecto D. Daniel Rubio Sánchez; el edificio del centro comercial Val General (1912) de D. Francisco Manuel Martínez 
Villena; la Plaza de Toros (1917) y el Instituto General y Técnico (actual Instituto del Bachiller Sabuco, 1923) de los arquitectos D. Julio Carrilero y D. Manuel Sáiz de Vicuña; el edificio Cabot (1922) del arquitecto D. Miguel Ortiz e Iribas; el Colegio Notarial (1923) de los arquitectos D. Manuel Muñoz Casayús y D. Julio Carrilero Prats y el Pasaje de Lodares (1925) del arquitecto D. Buenaventura Ferrando Castells.

\section{El automóvil en Albacete}

Las primeras referencias que se tienen respecto al automóvil en la ciudad de Albacete aparecen en la documentación fotográfica del Archivo Histórico Provincial de Albacete. El primer automóvil que llegó a Albacete (Figura 4) fue en 1905, propiedad de los actores D. Fernando Díaz de Mendoza y Aguado y Dạ. María Guerrero Torija, aunque en la misma la documentación gráfica se indica que previamente circuló por las calles de Albacete un monoplaza de motor y sin carrocería, diseñado, montado y conducido por D. Antonio Rodríguez Sánchez. Otro de los primeros datos es el que aparece en la documentación histórica de la Dirección General de Tráfico, donde se indica que el primer vehículo matriculado en Albacete fue un Charron tipo Sedán, propiedad de D. Ernesto Coloma Martínez de Almansa en 1908 (DGT, 1965).

\section{Figura 4. Primer automóvil en Albacete (1905)}

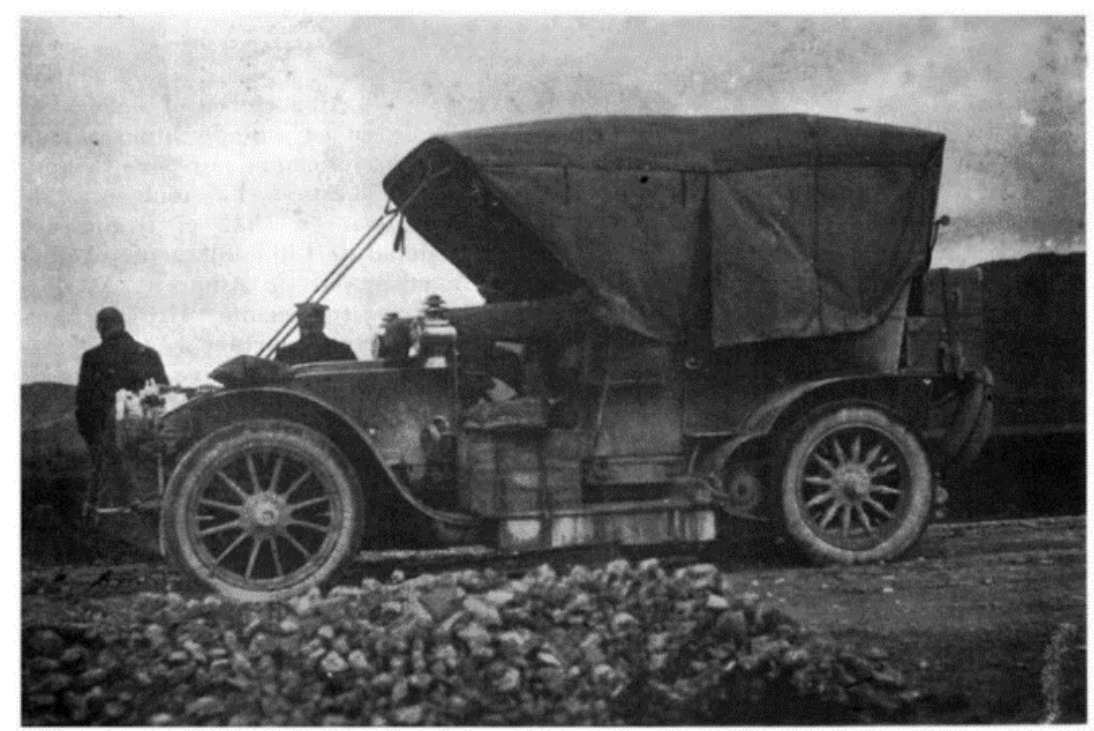

Fuente: AHPA.

El número de vehículos matriculados por año en el período 1900-1929 en Albacete se muestra en la Figura 5. Al igual que en el resto de las ciudades españolas la introducción entre los años 1915 y 1920 del "Ford T", vehículo orientado hacia la clase media, aumentó el número de adquisiciones y matriculaciones. Al tratarse de una ciudad pequeña, el número de matriculación de vehículos es mucho menor que en las grandes ciudades y esto también va de alguna manera influir en el desarrollo de las infraestructuras relacionadas con el automóvil, en especial en el nacimiento y desarrollo de los garajes. En Valencia el primer garaje data del año $1914^{1}$ y en Buenos Aires del año $1910^{2}$.

\footnotetext{
${ }^{1}$ Garaje Victoria, proyecto de D. Francisco Almenar.

${ }^{2}$ Garaje Centenario, proyecto D. Carlos Altgelt.
} 
Figura 5. Evolución del número de vehículos matriculados en la provincia de Albacete (1900-1929)

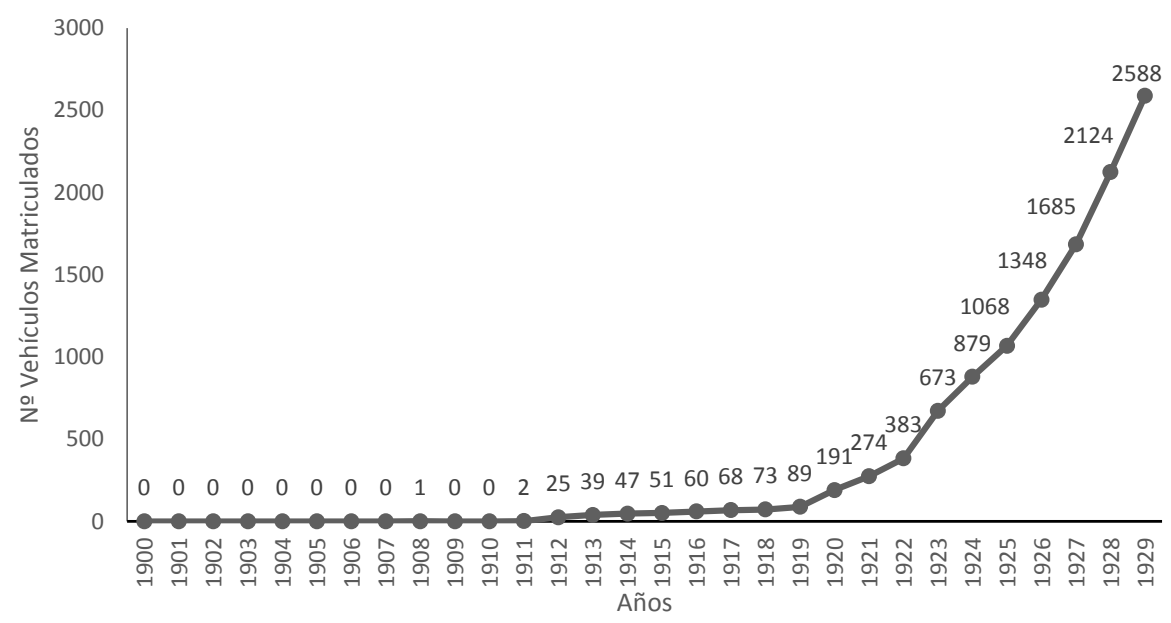

Fuente: Elaboración propia a partir de los datos de la Dirección General de Tráfico

El aumento del número de matriculaciones, y en consecuencia del número de ventas, se refleja también en el incremento de anuncios en los periódicos de la época (Figura 6). De hecho, en 1925, del 22 al 27 de septiembre, se celebró en el recinto ferial de Albacete la primera Feria de Automóviles de ocasión en un local cubierto y cerrado, organizada por el Ayuntamiento de Albacete, tal y como reza su anuncio en el periódico "El Defensor de Albacete" de fecha 11 de agosto de 1925.

Figura 6. Anuncios de agencias de ventas de automóviles en los diarios "El Defensor de Albacete" y "El Diario de Albacete"
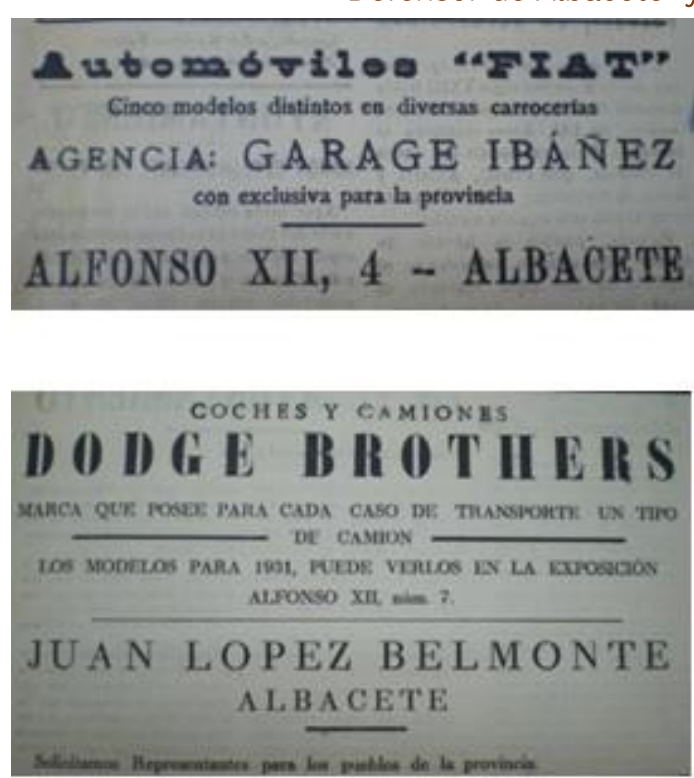

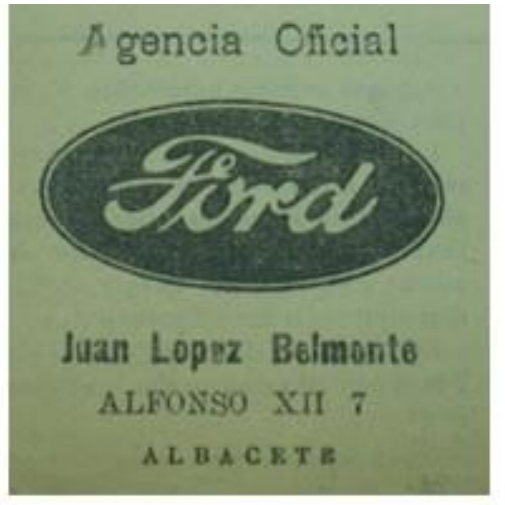

AขTom6vings

M A T IIIS

E ROLLS-ROYCB de las voiturettes : $x$ Recond del moado

de coniumo s: a,jso litros los too kllometron

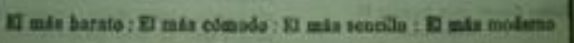

Jend Iraria Blaue.-Albseete

Fuente: AHPA.

El aumento del número de vehículos en la ciudad de Albacete y en la provincia a mitad de la década de los años 20 ya es un hecho, prueba de ello es la promulgación de unas normas de circulación por parte del gobernador civil de la provincia D. Alfonso de Lara el 1 de septiembre de 
19253. En estas normas se regulan aspectos tan diversos como la documentación del vehículo, la circulación por las calles estrechas de la ciudad, la velocidad en las carreteras de acceso a la ciudad, los ruidos o aspectos tan novedosos en la época como el espejo retrovisor, tal y como se cita en el punto cuarto de la regulación: "Los vehículos de motor mecánico destinados al transporte llevarán un espejo dispuesto en forma que permita que su conductor vea en todo momento a los que con mayor marcha caminen en su misma dirección, siendo obligatorio dejarles pase rápido e inmediato, conservando en su marcha la derecha....."

El automóvil se convierte poco a poco durante la década de los años veinte en algo cotidiano para la sociedad albaceteña, incorporándose a actos tan lúdicos e importantes como la Feria de Albacete, tal y como se puede comprobar en la Figura 7, donde se muestra la incorporación de los automóviles en las cabalgatas.

Figura 7. Imágenes de automóviles en la cabalgata de la Feria de Albacete
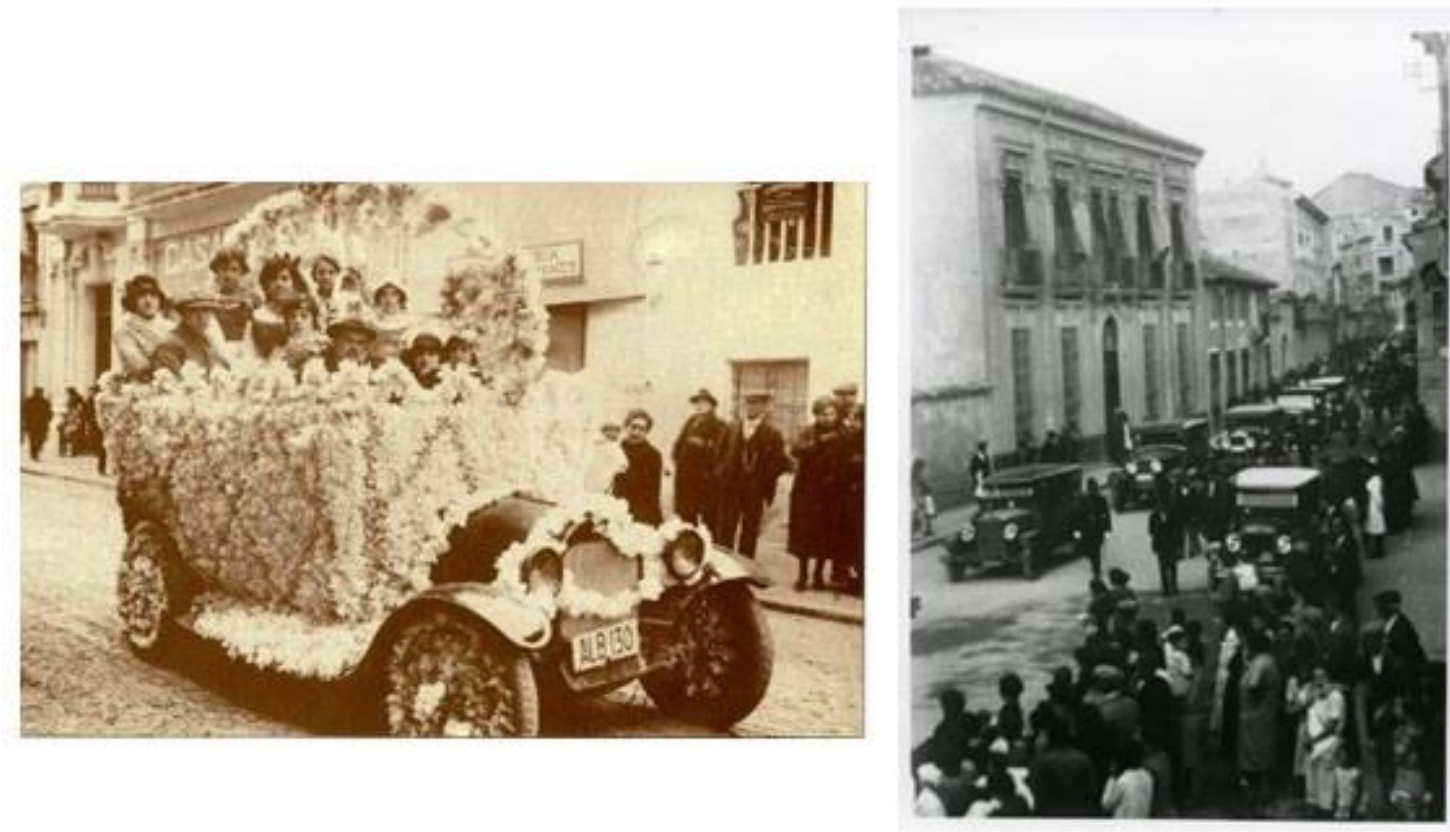

Fuente: AHPA.

\section{Los garajes y las estaciones de servicio}

En el año 1922 aparece en el Archivo Histórico Municipal de Albacete (AMA) la primera referencia de una solicitud de licencia de construcción de un edificio destinado a garaje (AMA. Caja 800. Fomento, Expediente 142). Se trata de una construcción diseñada por los arquitectos D. Julio Carrilero y D. Manuel Muñoz, promovida por D. Francisco Sánchez Collado en la calle del Tinte no 2 (Figura 8). Se trata de un garaje de $55 \mathrm{~m}^{2}$ para un camión donde se renuncia a edificar parte de la esquina del solar para facilitar el acceso del vehículo, ubicado justo en frente de la Posada del Rosario y en una de las principales vías de circulación de la ciudad de la época.

\footnotetext{
${ }^{3}$ AHPA. El Defensor de Albacete, miércoles 2 de septiembre de 1925.
} 


\section{ACE Architecture, City and Environment}

E-ISSN 1886-4805
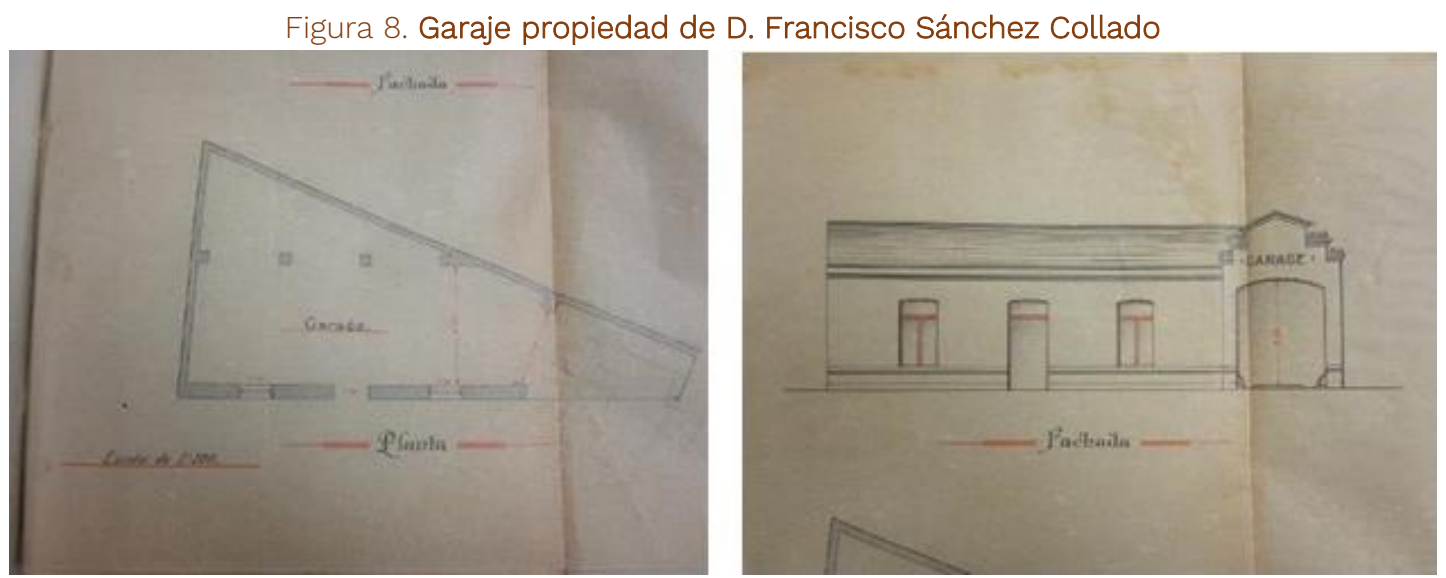

Fuente: AMA, Caja 800, Fomento Expediente 142.

En el año 1924, D. Juan López Belmonte solicita la construcción de una nave de nueva planta con destino a garaje (AMA, Caja 805, Fomento, Expediente 410) en una finca situada en el Barrio de la Industria, en la calle en proyecto (Figura 9). El proyecto está firmado en solitario por el arquitecto D. Julio Carrilero y se trata de una nave de $338 \mathrm{~m}^{2}$ sobre un solar de $1.400 \mathrm{~m}^{2}$. Este edificio se proyecta con diversos usos relacionados con el automóvil: un salón exposición en la fachada, una zona de taller en la parte posterior y entre ambas zonas se incluyen 34 cabinas individuales para garaje, junto a dos locales de WC para los usuarios y un depósito de gasolina.

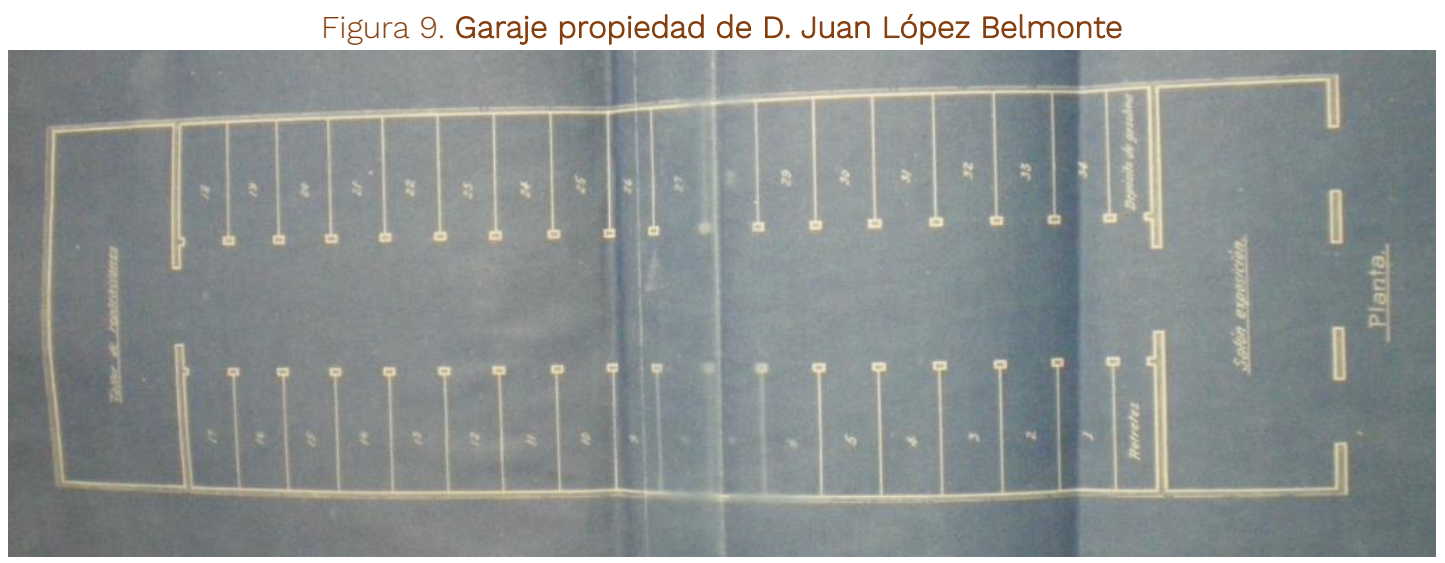

Fuente: AMA, Caja 805, Fomento, Expediente 410.

Justo diez días después de obtener la licencia, el 30-10-1924, el promotor solicita la visita del arquitecto municipal (en esta fecha D. Francisco Fernández Molina) con objeto de comprobación de la obra ejecutada y la devolución de parte de los arbitrios municipales, ya que la obra se había reducido porque la primitiva era excesiva para las necesidades del promotor (este dato muestra que la solicitud de la licencia municipal (05-09-1924) se hizo con la obra prácticamente finalizada). En la liquidación de arbitrios solicitada se indica que la superficie final del garaje fue de $171 \mathrm{~m}^{2}$, pero no se refleja planimetría de la edificación final.

En el año 1925, en el Paseo Alfonso XII no 4 (actualmente Paseo de la Libertad), que era el vial principal que llevaba hacia la antigua estación del ferrocarril, D. Estanislao Ibáñez Martínez solicitó licencia para la construcción de un garaje y sus dependencias (AMA, Caja 806, Fomento, Expediente 102). El proyecto, de $325 \mathrm{~m}^{2}$ en planta, fue redactado por el arquitecto D. Julio Carrilero, con un programa de necesidades, según reza en la memoria, de garaje, exposición y tienda (Figura 10). 


\section{Figura 10. Garaje propiedad de D. Estanislao Ibáñez Martínez}

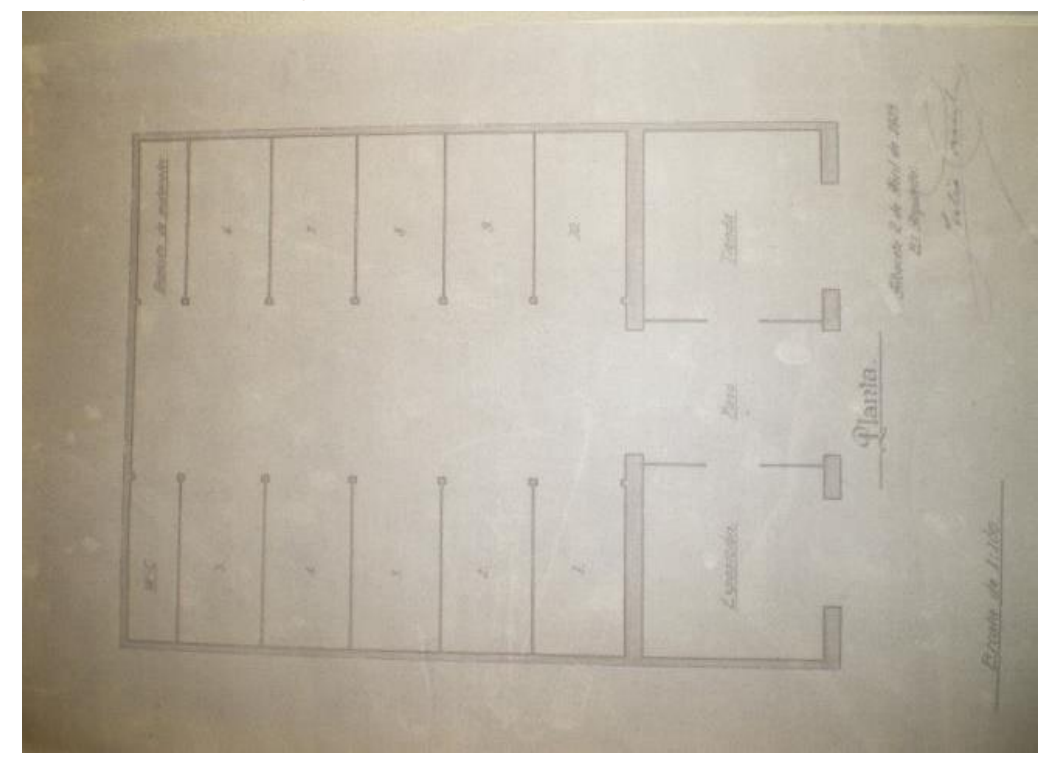

Fuente: AMA, Caja 806, Fomento, Expediente 102.

En 1928, D. Antonio Díaz González, en parte del solar de la esquina entre las calles Octavio Cuartero y Marques de Villores, promueve la construcción de un garaje (AMA, Caja 806, Fomento, Expediente 267) con cinco cabinas (Figura 11), con una superficie de $116,37 \mathrm{~m}^{2}$, diseño del arquitecto $D$. Francisco Fernández Molina.

Figura 11. Garaje propiedad de D. Antonio Díaz González
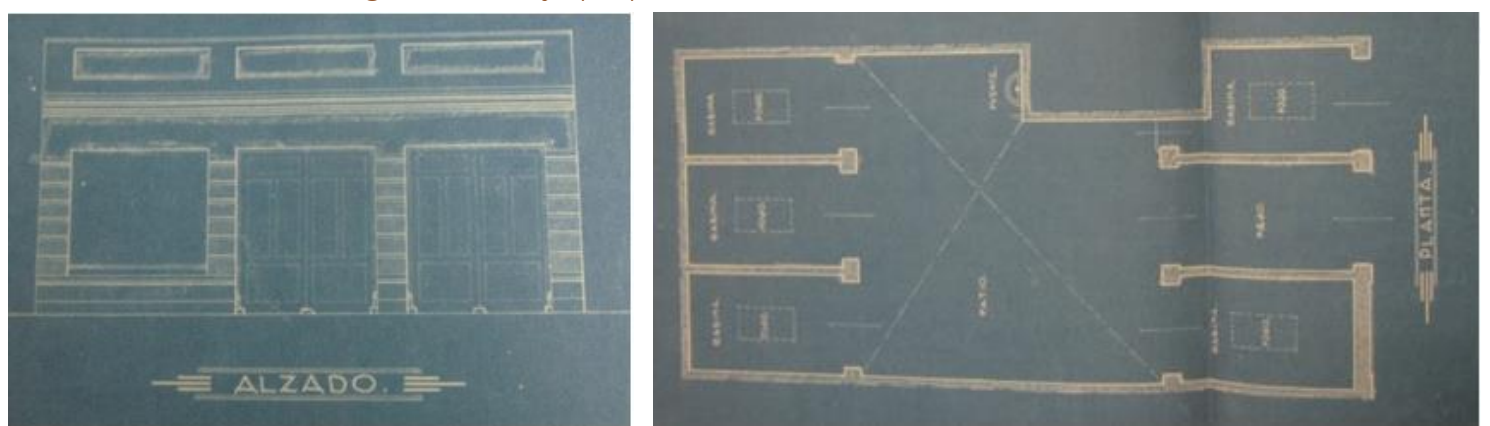

Fuente: AMA, Caja 806, Fomento, Expediente 267.

Se trata de un garaje con forma rectangular, con dos edificaciones y un patio central. En el edificio posterior se ubican tres cabinas y en el edificio principal se ubican las otras dos cabinas, teniendo una de ellas acceso individual desde la calle, mientras que el acceso al resto de las cabinas se realiza por la puerta situada en el hueco central de la fachada recayente a la calle Marqués de Villores. Las cabinas de este garaje presentan, a diferencia de los expedientes anteriores, un foso individual en cada una de las cabinas, lo que refleja que en este garaje se realizaban tareas de reparación y mantenimiento de los vehículos. La fachada principal presenta una simetría geométrica que se adapta a las características del solar, permitiendo disponer de una cabina con acceso independiente y funcionalmente se rompe la simetría dotando a la otra cabina de un ventanal que permite contemplar desde el exterior del garaje el vehículo aparcado en su interior, bien sea con una función comercial (venta) o de reconocimiento social, dada la ubicación del edificio en el centro de la ciudad. 
A partir de esta fecha y con el auge del vehículo en la sociedad burguesa albaceteña comienzan a proliferar las construcciones de pequeños garajes o cocheras para uno o dos vehículos a nivel particular, con o sin infraestructura de taller, tal y cómo se muestra en la relación de expedientes de la Tabla 1.

Tabla 1. Pequeños garajes en la ciudad de Albacete 1926-1929

\begin{tabular}{|l|l|l|l|l|l|}
\hline Promotor & Emplazamiento & Año & Arquitecto & Tipología & Expediente \\
\hline \hline $\begin{array}{l}\text { D. Ángel González } \\
\text { Sánchez }\end{array}$ & $\begin{array}{l}\text { C/ Marqués de } \\
\text { Villena }\end{array}$ & 1926 & $\begin{array}{l}\text { D. Buenaventura } \\
\text { Ferrando Castells }\end{array}$ & $\begin{array}{l}\text { Edificio de 2 } \\
\text { viviendas con } \\
\text { garaje en planta } \\
\text { baja }\end{array}$ & $\begin{array}{l}\text { Caja 807. Fomento. } \\
\text { Expediente. 74. }\end{array}$ \\
\hline $\begin{array}{l}\text { D. Gervasio } \\
\text { Fernández Martínez }\end{array}$ & $\begin{array}{l}\text { C/ de la } \\
\text { Industria }\end{array}$ & 1929 & $\begin{array}{l}\text { D. Francisco } \\
\text { Fernández Molina }\end{array}$ & $\begin{array}{l}\text { Garaje } \\
\text { individual + } \\
\text { Taller }\end{array}$ & $\begin{array}{l}\text { Caja 813. Fomento. } \\
\text { Expediente. 267. }\end{array}$ \\
\hline $\begin{array}{l}\text { Dá. Dolores } \\
\text { Belmonte Dumont }\end{array}$ & $\begin{array}{l}\text { C/ Teodoro } \\
\text { Camino }\end{array}$ & 1929 & $\begin{array}{l}\text { D. Francisco } \\
\text { Fernández Molina }\end{array}$ & $\begin{array}{l}\text { Garaje (2 } \\
\text { cabinas) }\end{array}$ & $\begin{array}{l}\text { Caja 813. Fomento. } \\
\text { Expediente. 226 }\end{array}$ \\
\hline $\begin{array}{l}\text { D. José María } \\
\text { Alcaraz Ortega }\end{array}$ & $\begin{array}{l}\text { C/ prolongación } \\
\text { de la calle de } \\
\text { los Condes de } \\
\text { Villarreal }\end{array}$ & 1929 & $\begin{array}{l}\text { D. Buenaventura } \\
\text { Ferrando Castells }\end{array}$ & $\begin{array}{l}\text { Garaje } \\
\text { individual }\end{array}$ & $\begin{array}{l}\text { Caja 813. Fomento. } \\
\text { Expediente. 90 }\end{array}$ \\
\hline $\begin{array}{l}\text { D. Emilio Belmonte } \\
\text { Dumont }\end{array}$ & $\begin{array}{l}\text { C/ Teodoro } \\
\text { Camino no 6 }\end{array}$ & 1929 & $\begin{array}{l}\text { Caja 814. } \\
\text { Fernández Molina }\end{array}$ & $\begin{array}{l}\text { Garaje (2 } \\
\text { cabinas) }\end{array}$ & Fomento. \\
Expediente. 359 \\
\hline
\end{tabular}

Fuente: AMA.

Además de los garajes y cocheras para uso individual, hay que destacar varios expedientes que reflejan la importancia del automóvil y las necesidades que genera en la ciudad de Albacete a finales de los años 20 e inicio de los años 30. D. Alfredo Moreno García promueve en 1929 un garaje AMA, Caja 812, Fomento, Expediente 295) con casa para chóferes en la carretera de Ocaña-Alicante, $\mathrm{Km} 243 \mathrm{Hm} 3$ (Figura 12). Se trata de una edificación de 2.219,5 m² ubicada a orillas de la carretera y destinada a la función de fonda y posada.

El complejo está diseñado por el arquitecto D. Julio Carrilero y está compuesto por un edificio de dos plantas con dos naves adosadas en su parte posterior. El edificio principal, con un frente de fachada de $33 \mathrm{~m}$, presenta dos plantas, la planta baja destinada a talleres y para el acceso a las naves donde se ubican los garajes, mientras que en la planta primera se ubican los dormitorios y aseos para los chóferes.

Las naves posteriores, presentan diferentes características, la nave de la izquierda está compuesta por dos zonas laterales con cubierta a un agua donde se ubican catorce cabinas individuales, a las que se accede por el patio central. En la nave de la derecha, con cubierta a dos aguas, se ubica un garaje sin división interior, rematado en la parte posterior con un patio descubierto. Esta división puede deberse simplemente a una ordenación de vehículos más pequeños (automóviles) en una nave y de vehículos de mayor tamaño (camiones o autobuses) en la otra nave, o tratarse de una división más relacionada con el poder adquisitivo de los propietarios de los vehículos.

En 1929, D. Francisco López Tendero, en representación de D. Eduardo Quijada Pérez, solicita la construcción en un solar de la calle Fontecha, ubicado entre el Banco Central y una vivienda particular, de un garaje (AMA, Caja 814, Fomento, Expediente 698) compuesto por ocho cabinas individuales con acceso independiente cada una de ellas (Figura 13). Se trata de un proyecto de gran sencillez del arquitecto D. Miguel Ortiz e Iribas, pero enfocado a una gran funcionalidad, dada su ubicación en pleno centro de la ciudad, que permite tanto el aparcamiento de propietarios de 
inmuebles cercanos como el aparcamiento de gente de paso o de negocios en la ciudad. Con la misma función y también en el centro de Albacete (calle Isaac Peral № 1), en el año 1930 solicita D. Gregorio Díaz Marta la realización de una cubierta a teja-vana para cinco cocheras (siendo el proyecto de D. Miguel Ortiz e Iribas).

Figura 12. Garaje propiedad de D. Alfredo Moreno García
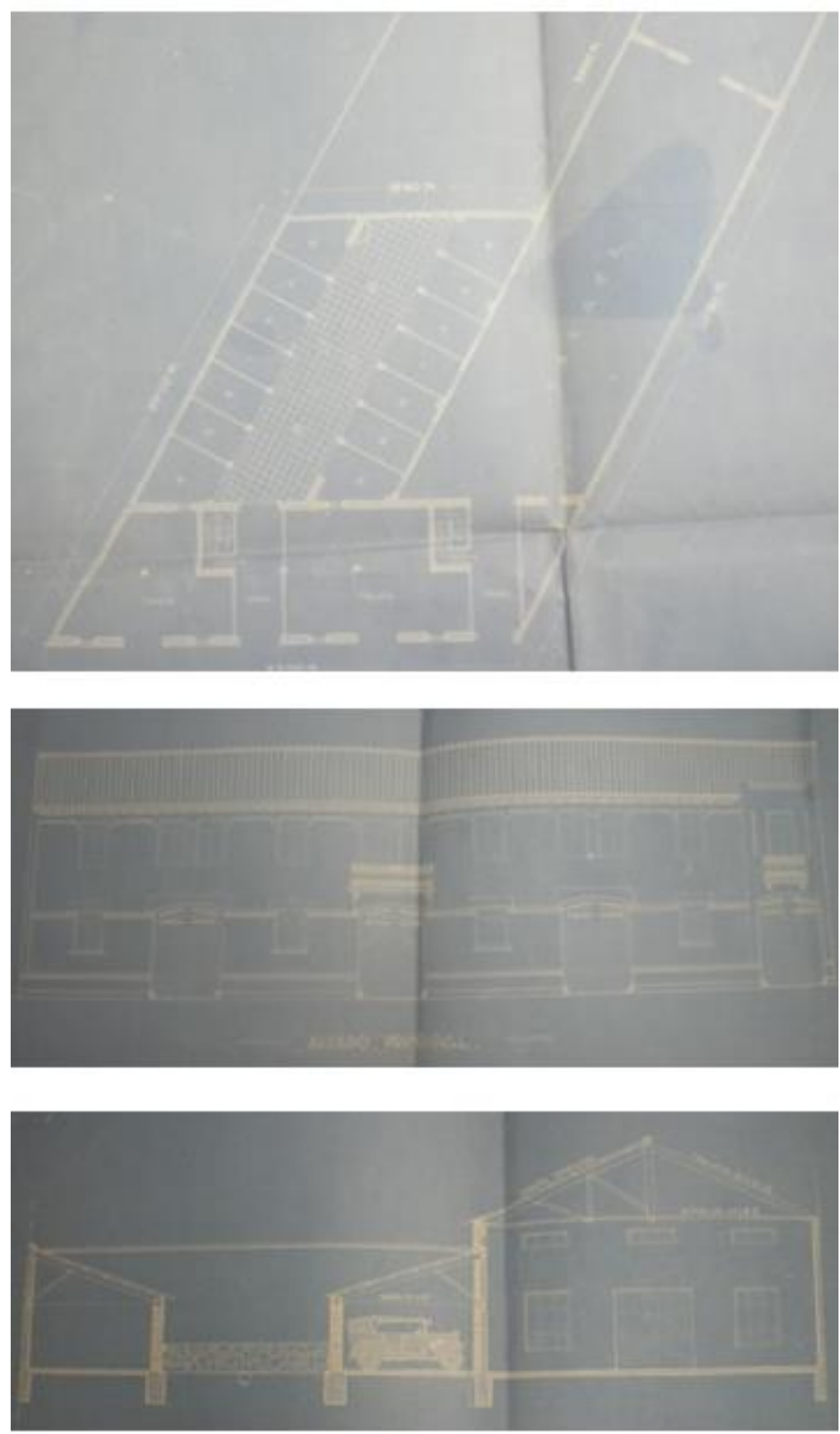

Fuente: AMA, Caja 812, Fomento, Expediente 295. 
Figura 13. Garaje propiedad de D. Francisco López Tendero
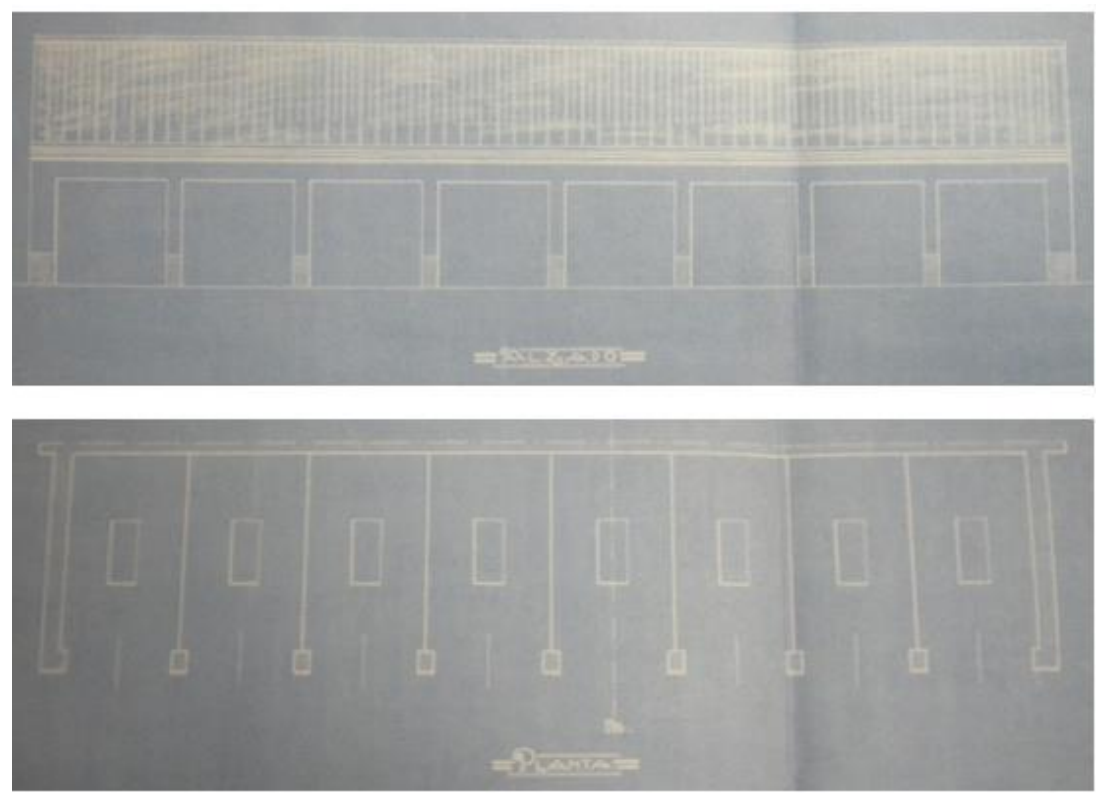

Fuente: AMA, Caja 814, Fomento, Expediente 698.

El año 1929 supuso también el desarrollo de otras infraestructuras indispensables para la consolidación del automóvil, el sistema de suministro de gasolina. La Compañía Arrendataria del Monopolio de Petróleos S.A. (CAMPSA) solicitó en unos terrenos próximos a la estación del ferrocarril la construcción de un almacenamiento a granel (AMA, Caja 813, Fomento, Expediente 89) que permitiera la correcta distribución de gasolina y productos pesados, tanto a la ciudad de Albacete como al resto de poblaciones de la provincia.

Figura 14. Distribución en planta de zona de almacenamiento de gasolina y productos pesados ${ }^{4}$

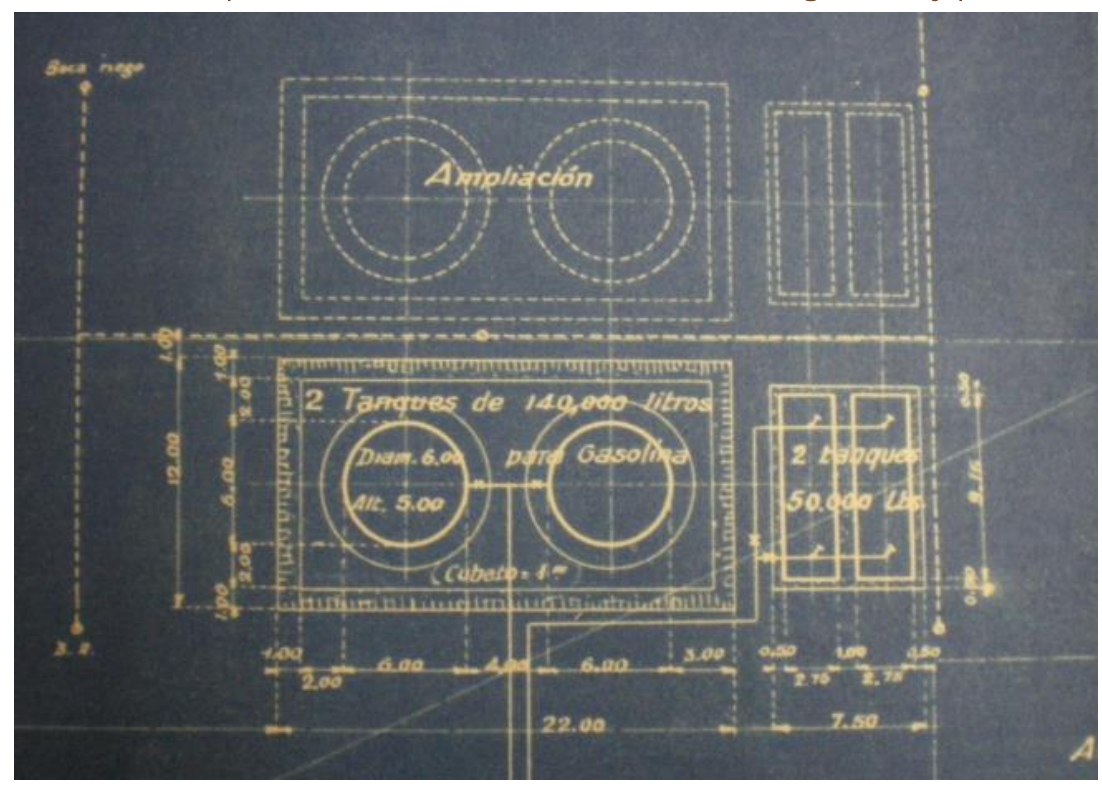

Fuente: AMA, Caja 813, Fomento, Expediente 89.

\footnotetext{
${ }^{4}$ El proyecto está firmado por Ingeniero Industrial de Madrid, no se indica el nombre en el expediente, solo aparece la firma.
} 
Estas instalaciones se ubicaron sobre una superficie de $8.608 \mathrm{~m}^{2}$, donde junto a las oficinas, garaje e instalaciones auxiliares necesarias para la carga y descarga de los vagones y camiones cisterna se instalaron dos depósitos de 140.000 litros para el almacenamiento de gasolina y dos tanques de 50.000 litros para productos pesados (Figura 14).

La instalación de la subsidiaria de CAMPSA supuso el inicio del negocio de la distribución del carburante en la provincia de Albacete. La empresa Hijos de José Legorburo se convirtieron en los delegados para la provincia de Albacete de CAMPSA y solicitaron la instalación de aparatos surtidores, por primera vez sin estar asociados a garajes, y siendo así la primera aproximación de las estaciones de servicio actuales. Esta empresa solicitó la instalación de un aparato surtidor y un depósito enterrado (AMA, Caja 813, Fomento, Expediente 307) con una capacidad de 7.500 litros en la población de Pozo Cañada ${ }^{5}$ (en la travesía de la carretera de Albacete a Cartagena) y otro aparato surtidor (AMA, Caja 814, Fomento, Expediente 729) en la carretera de Ocaña a Alicante, en las cercanías del garaje de D. Alfredo Moreno (Figura 15).

Figura 15. Distribución en planta surtidor de Pozo Cañada y Sección de surtidor de Albacete ${ }^{6}$
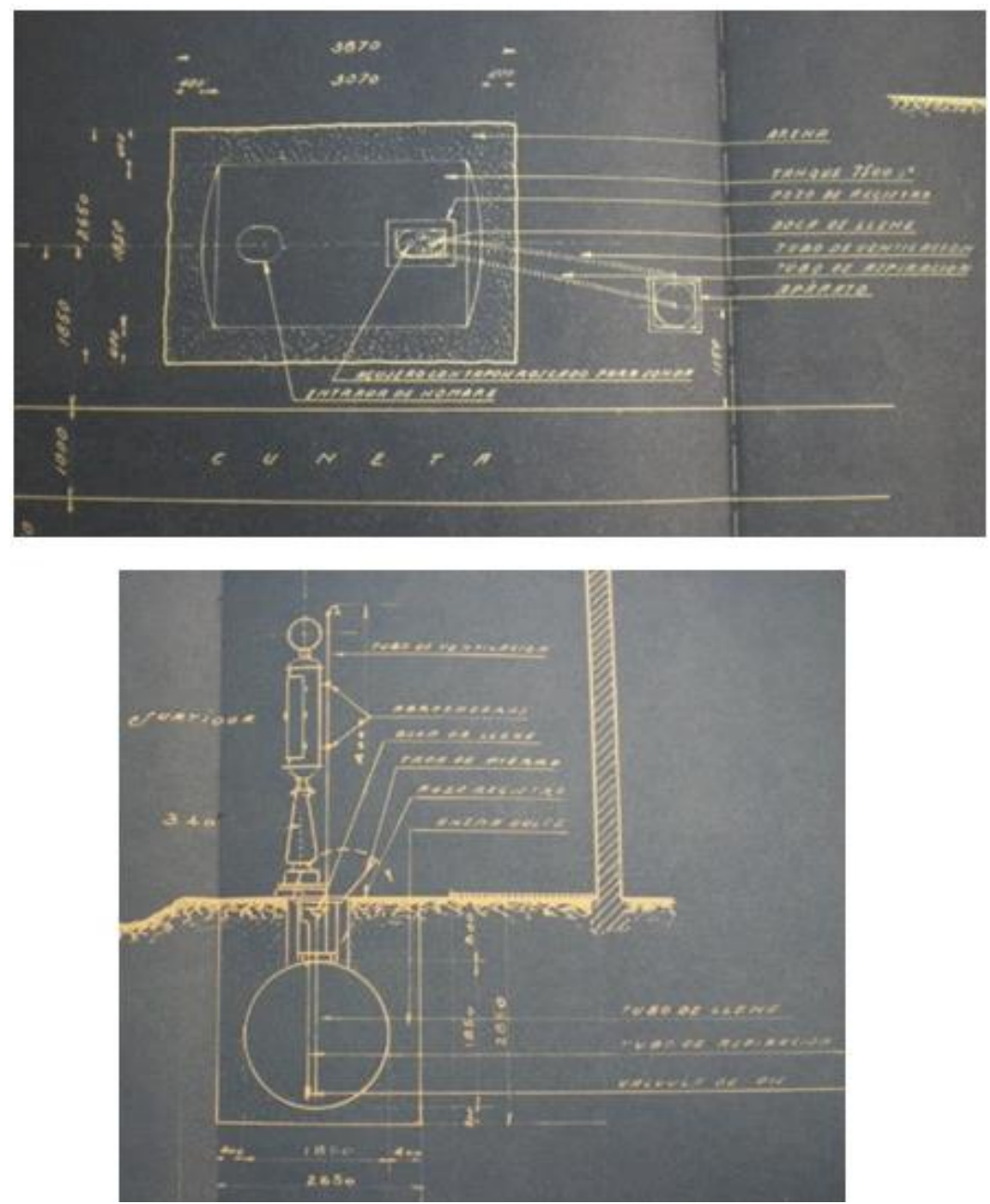

Fuente: AMA, Caja 813, Fomento, Expediente 307 y Caja 814, Fomento, Expediente 729.

\footnotetext{
${ }^{5}$ En la fecha de estudio, la población de Pozo Cañada era una pedanía de la ciudad de Albacete, dependiendo todas las solicitudes de licencia de obra del Ayuntamiento de Albacete.

${ }^{6}$ El proyecto está firmado por Ingeniero Industrial de Madrid, no se indica el nombre en el expediente, solo aparece la firma.
} 
Como punto final del estudio se ha de señalar el expediente de construcción de casa de nueva planta de D. Francisco Sedano Lasuen de 1930 en la calle García Más. En el proyecto de D. Julio Carrilero se plantea un edificio de planta baja y planta primera y dos naves interiores, con un programa de necesidades muy definido en la memoria del proyecto (Figura 16).

Figura 16. Casa y Garaje de D. Francisco Sedano Lausen
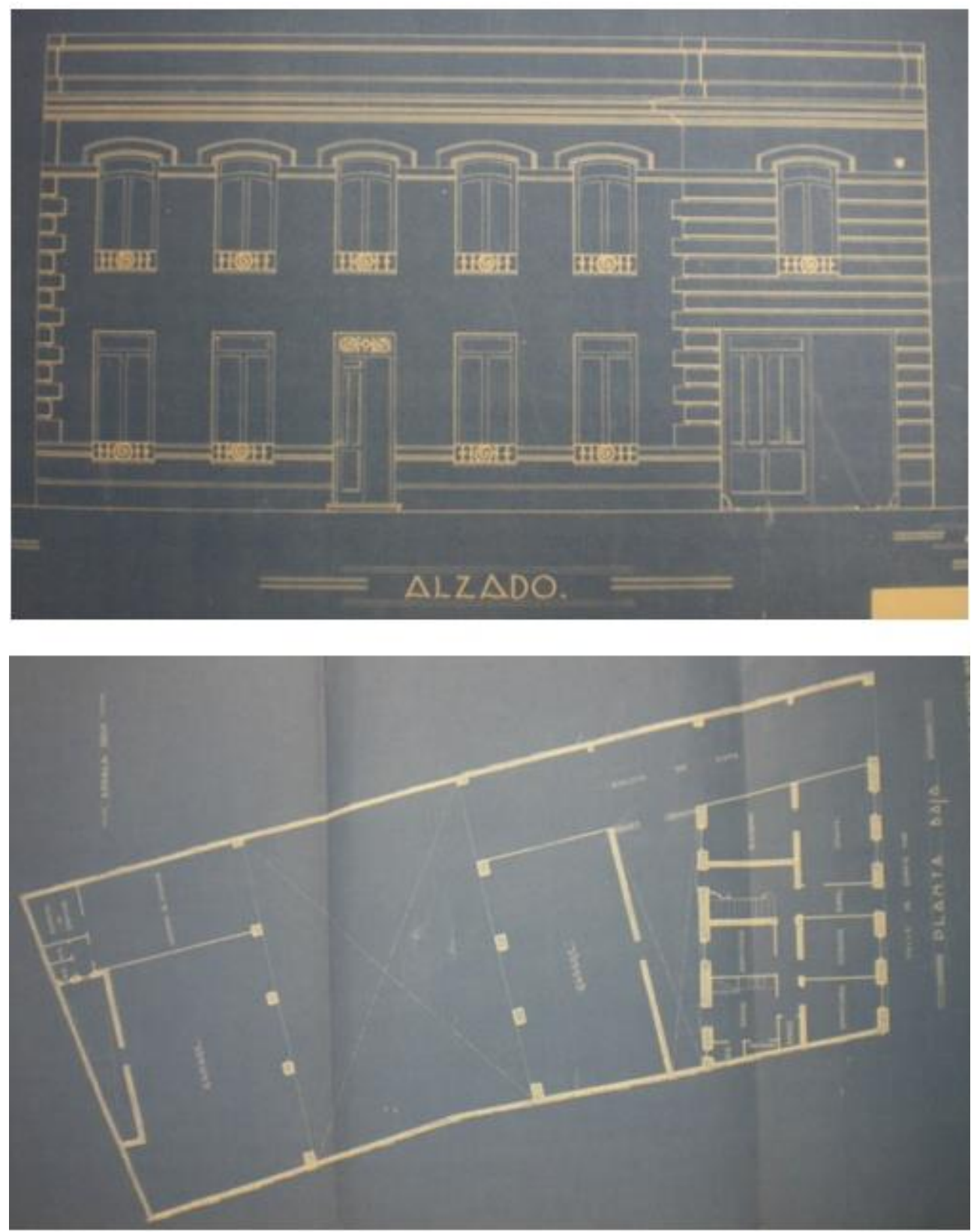

Fuente: AMA, Caja 815, Fomento, Expediente 79.

La planta baja estaba destinada para oficinas y vivienda del guarda, las naves interiores destinadas a garaje (AMA, Caja 815, Fomento, Expediente 79) y lavadero de vehículos y las viviendas superiores destinadas a viviendas para empleados. 


\section{La arquitectura de los garajes y de las estaciones de servicio}

\subsection{Las normas}

Las condiciones de diseño y de construcción de las infraestructuras del automóvil en la ciudad de Albacete se desarrollaron a través de las Ordenanzas Municipales del 1 de septiembre de 1904, redactadas por el secretario del Ayuntamiento de la época, D. Joaquín Quijada Valdivieso, y más concretamente en el título 10 donde se reflejan los requisitos relativos a las construcciones.

En el capítulo 3 del citado Título 10 de las Ordenanzas se reordena la altura de las edificaciones en función de la anchura de los viales, si bien todos los garajes del período de estudio se ubican en edificios de planta baja o de planta baja más planta primera.

Respecto de los aparatos surtidores, en los proyectos de su instalación no se hace referencia a ninguna norma en vigor, pero sí que presentan una estructura de proyecto muy definida: una memoria tipo (Figura 17) donde únicamente se modifican los campos correspondientes a la titularidad del aparato, el emplazamiento y la capacidad del depósito, y una planimetría donde se refleja el emplazamiento, la distribución en planta y los detalles de sección del aparato surtidor y del depósito. En la memoria se describen las características de los depósitos, las condiciones de cubrición, la tipología y las características del aparato surtidor y las conexiones entre éste y el depósito.

Figura 17. Memoria del proyecto de Aparato Surtidor en Pozo Cañada
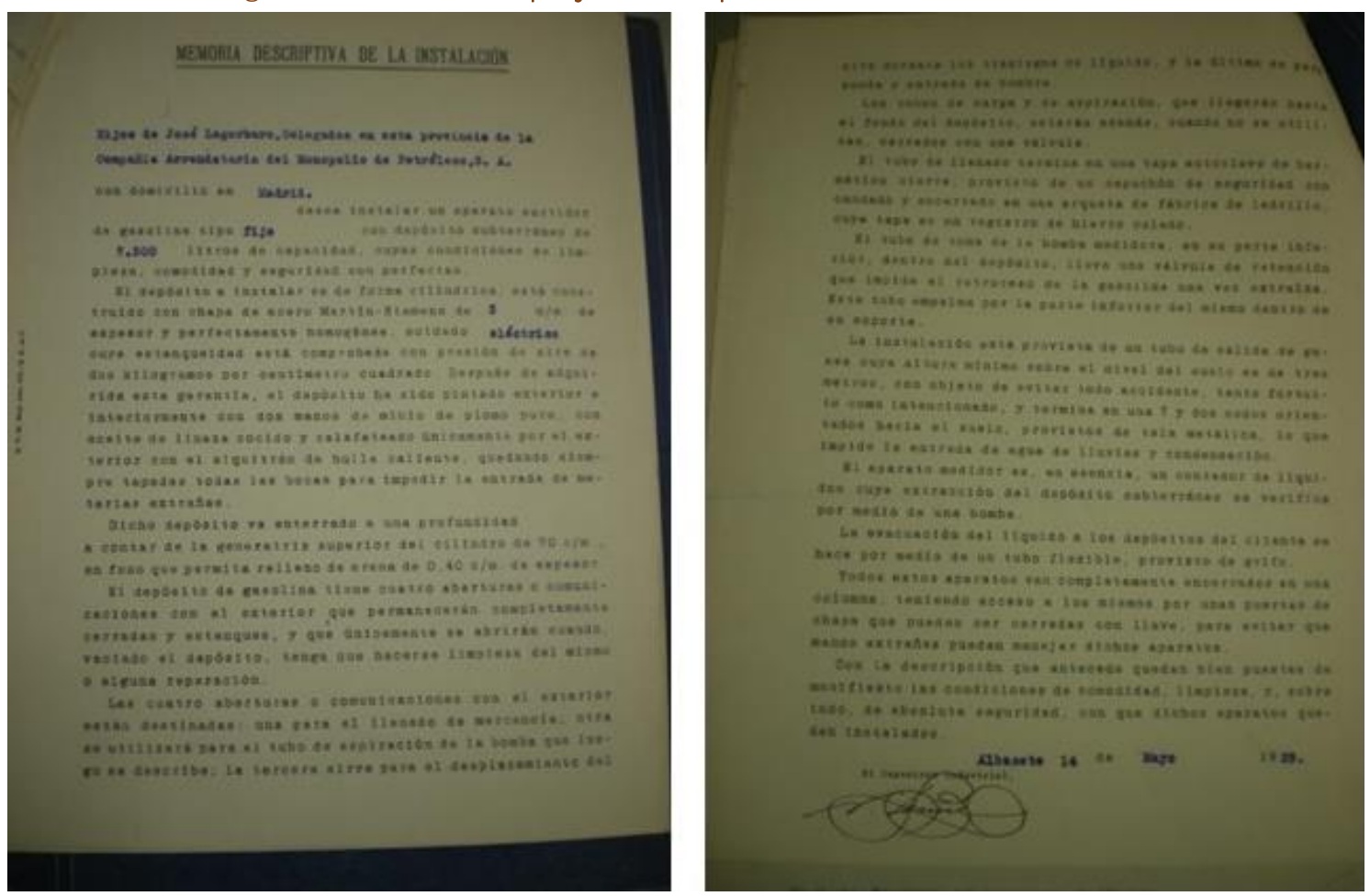

Fuente: AMA, Caja 813, Fomento, Expediente 307. 


\subsection{El programa de necesidades}

En el diseño de cualquier edificación, el programa de necesidades determina las características que debe cumplir el edificio según las exigencias del promotor y de los requisitos técnicos y normativos. En los garajes identificados en la ciudad de Albacete se pueden identificar dos grupos, por un lado, los edificios que expresamente se crean para las actividades relacionadas con el automóvil y por el otro lado aquellos cuyo objetivo es disponer de espacio para aparcamiento para automóviles propios o de terceros, o incluso como valor añadido en edificios destinadas a viviendas de alquiler.

En el primer grupo se pueden incluir los garajes de D. Juan López Belmonte, D. Estanislao Ibáñez, D. Antonio Díaz, D. Alfredo Moreno y D. Francisco Sedano. En estos edificios se destina una zona a taller de reparación y otra zona para aparcamiento mediante cabinas, con unas dimensiones aproximadas entre 5 y $6 \mathrm{~m}$ de longitud y entre 2,5 y $3 \mathrm{~m}$ de ancho.

Además de las funciones básicas de los garajes también se desarrollaron otras actividades complementarias como la venta de vehículos (Garaje López Belmonte, Figura 9, y Garaje Ibáñez, Figura 10) y de accesorios (Garaje Ibáñez), siendo quizás estas dos instalaciones las que más semejanza tienen con garajes de otras ciudades de mayor tamaño.

El garaje López Belmonte y el garaje Sedano disponen también de un aparato surtidor de gasolina en el interior de la instalación, lo que proporciona un valor añadido a los usuarios del garaje, dada su escasez en la ciudad y en la provincia, ya que la proliferación de los aparatos surtidores se realizó a partir de la instalación de CAMPSA en la ciudad de Albacete en el año 1929. Otra de las actividades complementarias de estas instalaciones fueron los lavaderos. El garaje Sedano (Figura 16) es el único que dispone de una zona identificada como tal en la planimetría.

El segundo grupo de garajes lo conforman el resto de las instalaciones identificadas en la Tabla 1 y los garajes de D. Francisco Sánchez y D. Francisco López Tendero. Resulta interesante resaltar dentro de este grupo dos casos. El primero de ellos es el garaje de D. Francisco López Tendero (Figura 13), el cuál presenta únicamente zonas de aparcamiento (mediante cabinas), adaptando la configuración del edificio a aprovechar al máximo la superficie del solar y cuyo principal objetivo, dada su ubicación, sería el de disponer de plazas de aparcamiento para alquiler.

El segundo caso es el edificio de viviendas para alquiler promovido por D. Ángel González en el que el arquitecto D. Buenaventura Ferrando Castells, en un solar pentagonal diseña un edificio con dos viviendas en planta primera a los que les asocia la posibilidad de un garaje o almacén en la planta baja (Figura 18).

En la Figura 19 se presenta un plano de Albacete con la ubicación de las diferentes instalaciones identificadas en el estudio (a excepción de los depósitos de CAMPSA y del aparato surtidos de Pozo Cañada). Se señalan con un punto negro aquellas instalaciones cuya ubicación viene definida en los expedientes de construcción y con un punto rojo las ubicaciones aproximadas de las instalaciones cuya ubicación no es tan precisa en los expedientes de construcción.

Se puede comprobar cómo los edificios que expresamente se crean para las actividades relacionadas con el automóvil se articulan en dos zonas: el centro de la ciudad cercano al Altozano y el actual Paseo de la Libertad (garajes Ibáñez, Díaz y López Tendero) y en torno a las carreteras de Ocaña a Alicante y la carretera de Jaén, punto de encuentro entre las carreteras que pasaban por la ciudad (garajes López Belmonte, Moreno y Sedano). 


\section{ACE Architecture, City and Environment}

Figura 18. Distribución en planta y Alzados de Edificio de Proyecto de casa de alquiler de D. Angel González
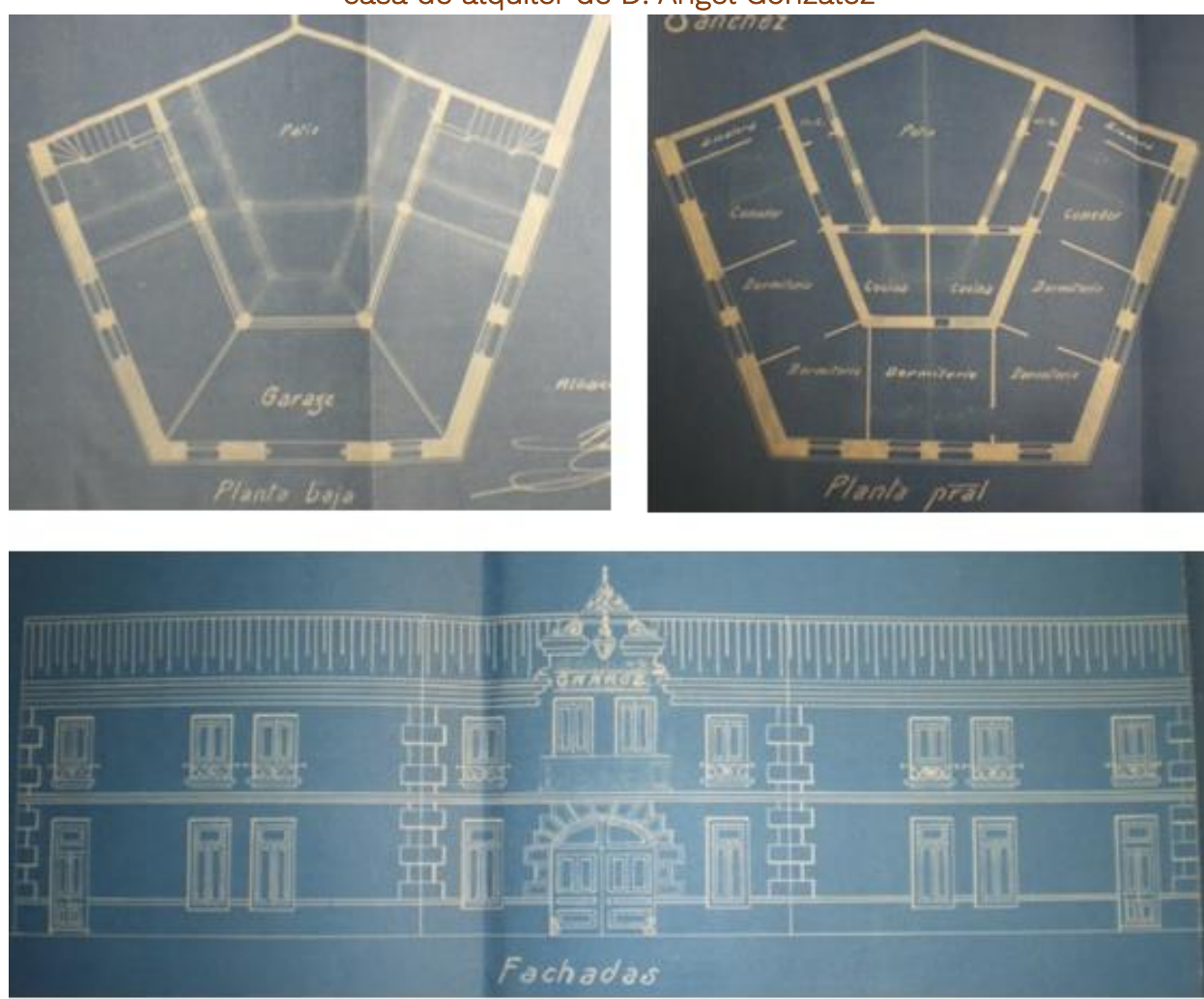

Fuente: AMA, Caja 807, Fomento, Expediente 74.

Figura 19. Emplazamiento de las instalaciones

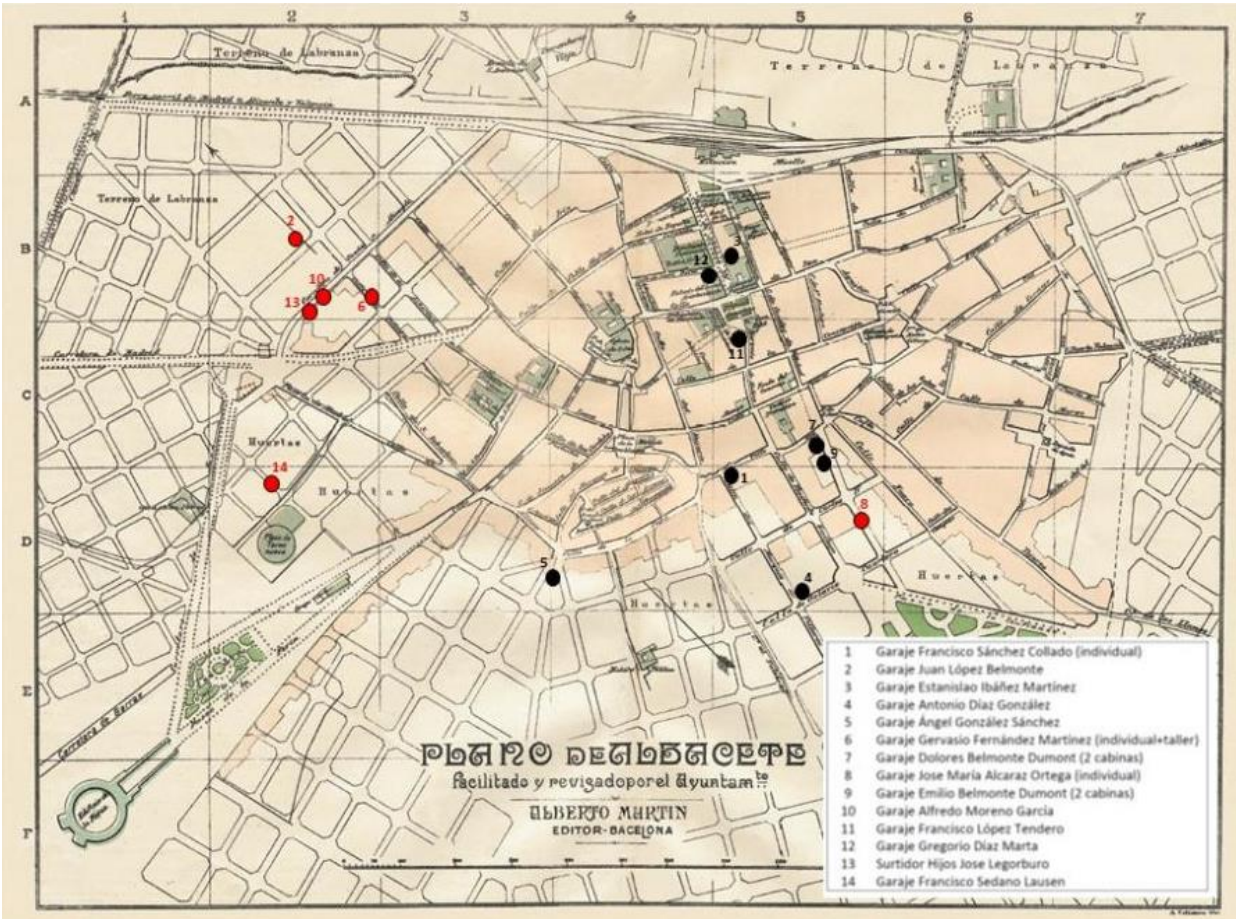

Fuente: Elaboración propia sobre plano de Albacete de 1915 


\subsection{Los materiales}

A diferencia de lo que sucede en un estudio similar realizado en la ciudad de Valencia (FuentesBargues, 2014), no quedan vestigios de las edificaciones citadas en este estudio ni tampoco que en los mismos emplazamientos (en edificaciones posteriores o con reformas de los edificios iniciales) se hayan mantenido los usos de garajes o agencias de venta de vehículos, por lo que el estudio de las tipologías constructivas y materiales empleados se refiere a la documentación escrita y gráfica de los expedientes de obra.

El sistema constructivo habitual durante la época en la ciudad de Albacete consistía en una cimentación a base de hormigón hidráulico y unos muros de cerramiento (en la mayoría de las edificaciones realizaban también funciones de muros de carga), a base de fábrica mixta de mampostería y tapial calicostrado.

En el aspecto referente a las cubiertas se distinguen diferentes tipos de cubierta: planas (como la de la nave trasera del garaje Díaz), inclinadas a un agua (la nave delantera del garaje Díaz, la zona de cabinas del garaje Moreno o del garaje López Tendero) o inclinadas a dos aguas.

En este último grupo hay que diferenciar entre los garajes con una o dos plazas de aparcamiento y los garajes de mayor capacidad. En los primeros se presenta una única nave con una cercha tipo Queen, a base de madera en los elementos principales (pares, montantes y diagonales) y de acero en los tirantes y en los elementos de unión. En los garajes Ibáñez y López Belmonte se presenta una tipología constructiva propia de los garajes de la época (Benito Goerlich 1992; Fuentes-Bargues 2014) formada por tres naves adosadas, siendo la nave central por donde circulan los vehículos y las naves laterales adosadas donde se encuentran las cabinas de aparcamiento, si bien presentan sus peculiaridades.

En el garaje López Belmonte (Figura 20) las tres naves tenían la misma altura y las tres con cubierta a dos aguas, conformada su estructura de cubierta mediante cerchas mixtas tipo Queen que apoyaban sobre soportes de "cemento". La nave central presentaba una linterna para dotar de ventilación e iluminación natural a la zona de circulación de vehículos.

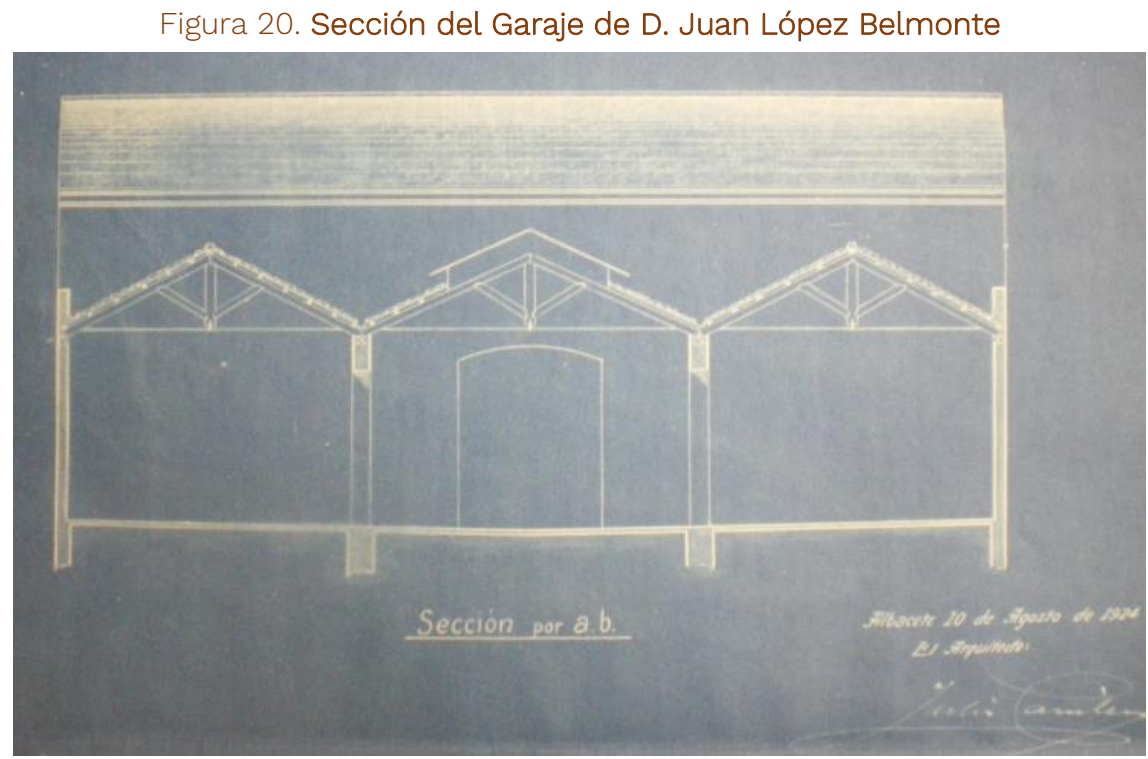

Fuente: AMA, Caja 805, Expediente 410. 
El garaje Ibáñez (Figura 21) presentaba tres naves, la central con mayor altura y una cubierta a dos aguas y las dos naves laterales con cubierta a un agua hacia canales exteriores. La diferencia de altura de la nave central se aprovechaba a modo de claristorio, para dotar de iluminación y ventilación a todo el garaje.

Figura 21. Sección del Garaje de D. Estanislao Ibáñez Martínez

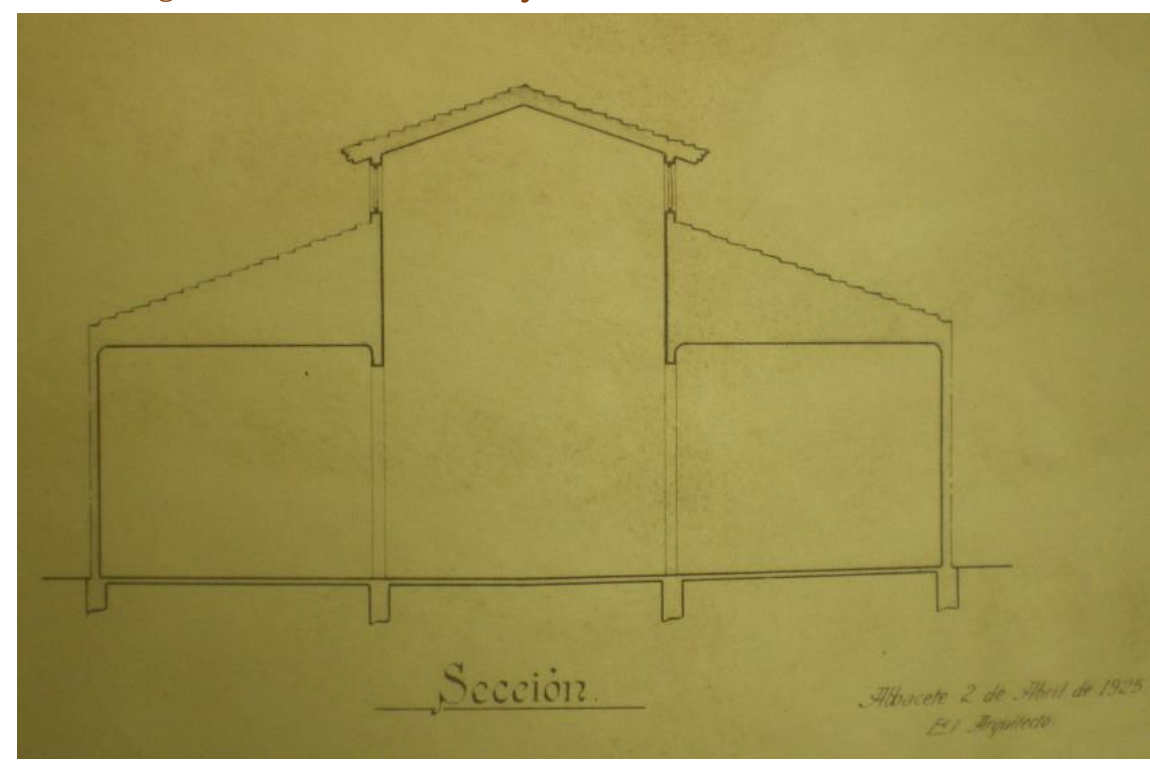

Fuente: AMA, Caja 806, Expediente 102.

Las tabiquerías interiores que dividían las cabinas o las estancias tales como despachos, zonas de exposición, venta de accesorios, etc., se realizaban mediante fábricas de ladrillo cerámico hueco. Los fosos para los trabajos de mantenimiento y reparación de los vehículos se realizaron mediante muretes de ladrillo guarnecidos con mortero de cemento sobre una base de hormigón.

\subsection{Las Fachadas}

Los garajes, como extensión del automóvil, se convierten en todas las ciudades como una representación visible de modernidad dentro de la sociedad de principios del siglo XX, siendo las fachadas su elemento más representativo, dónde los arquitectos plasman las tendencias de diseño de las corrientes arquitectónicas de la época (Fuentes-Bargues, 2014; Muñoz, 2018).

En este aspecto resultan destacables las fachadas de dos de los primeros garajes que se identifican en el período de estudio, ambos proyectos firmados por el arquitecto D. Julio Carrilero, el garaje López Belmonte y el garaje Ibáñez, donde el arquitecto pone de manifiesto su estilo modernista con gran maestría.

Respecto al garaje López Belmonte se desconoce si la modificación del proyecto afectó a la originalidad y vistosidad de la fachada del proyecto original (Figura 22). Se trata de una composición simétrica de tres cuerpos. En el cuerpo central se ubica el hueco de la puerta de acceso a la zona de garaje, de líneas rectas y de mayor tamaño que los huecos laterales, los cuales, realizados también con formas rectas, permitían el acceso a la sala de exposición tanto de personas como de vehículos. Los dos cuerpos laterales simulan mediante almohadillado unas pilastras que sostienen al cuerpo central, coronado éste por una composición formada por un dintel que es soportado por dos pináculos decorados con pequeñas volutas, en armonía con las volutas de mayor tamaño que conexionan la parte superior de los tres cuerpos que conforman la fachada. 


\section{ACE Architecture, City and Environment}

E-ISSN $1886-4805$

Figura 22. Fachada del Garaje de D. Juan López Belmonte

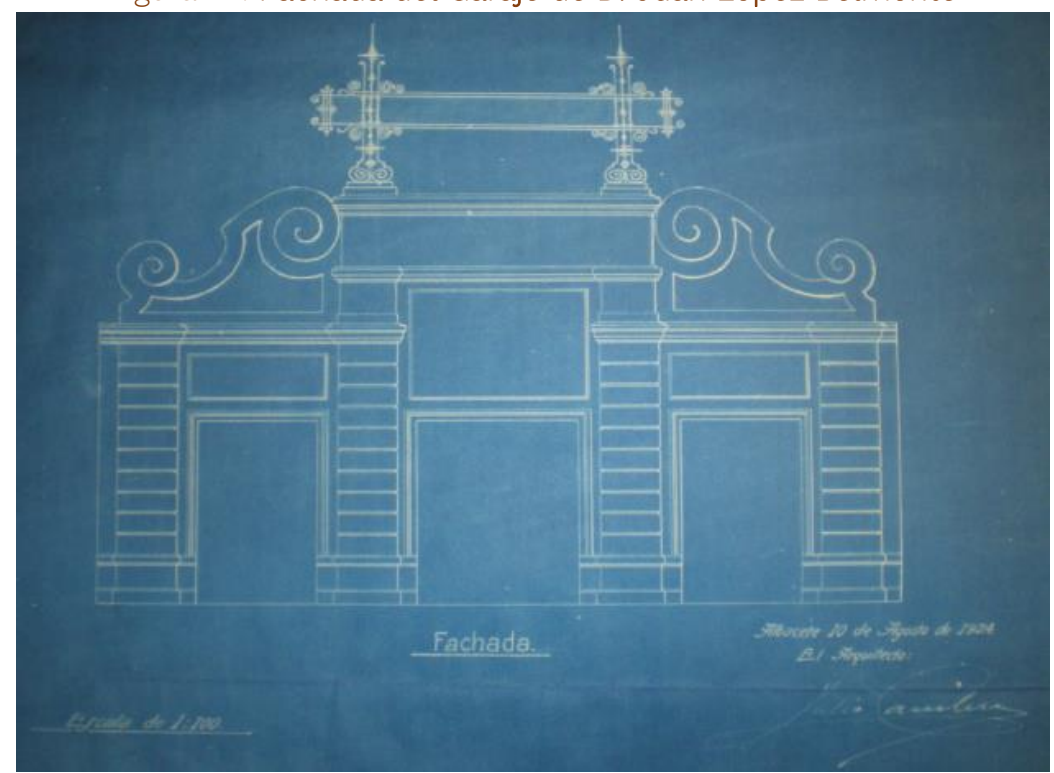

Fuente: AMA, Caja 805, Expediente 410.

El garaje Ibáñez presentaba una fachada simétrica compuesta por tres huecos (Figura 23) Los huecos laterales permitían el acceso a la tienda y al almacén y estaban realizados con líneas rectas y un almohadillado en el dintel superior ocupando la mitad de su longitud. La nave central presenta una doble altura, ubicándose en la parte inferior el acceso de vehículos a través de un hueco de puerta realizado mediante un arco rebajado. Este hueco se decoraba resaltando la clave mediante arcos decorativos yuxtapuestos que se apoyan sobre unos capiteles, que recordaban de algún modo al orden jónico. En la doble altura se presentaba un marco central remarcado por dos pilastras de almohadillados que descansaban sobre unos capiteles, en este caso menos recargados. Como coronación del marco central se presentaba un frontón rebajado, ornamentado mediante volutas y motivos florales en el que centro se mostraba el esbozo de un escudo heráldico.

Figura 23. Fachada del Garaje de D. Estanislao Ibáñez Martínez

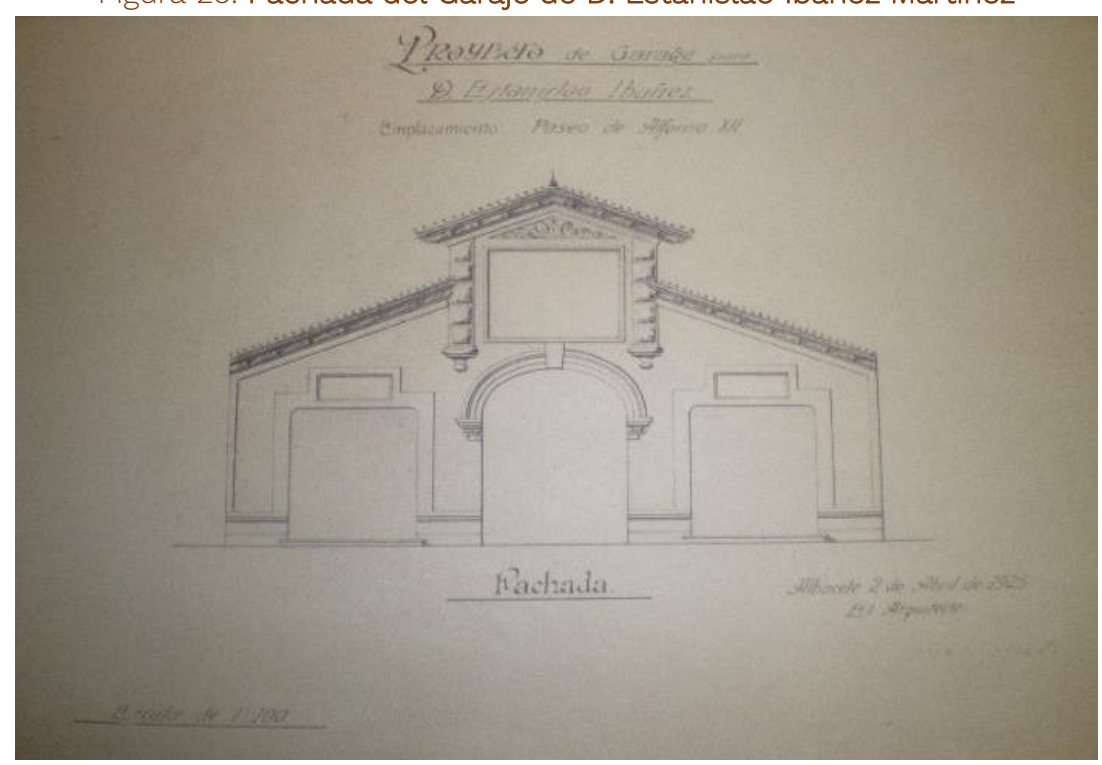

Fuente: AMA, Caja 806, Expediente 102.

ACE, 16 (4.6) CC BY-ND 3.0 ES | UPC Barcelona, España | Título del artículo escrito en mayúsculas y minúsculas, si 


\subsection{Los arquitectos}

Tal y como se ha señalado en la identificación y descripción de cada uno de los garajes, los diseños de los garajes proceden de los principales arquitectos de la época en la ciudad de Albacete. El más prolífico en número de obras es D. Julio Carrilero, que presenta cuatro proyectos en solitario y uno con su socio D. Manuel Múñoz. La magnífica capacidad y trayectoria de Julio Carrilero en la ciudad de Albacete y en especial en el período de estudio nos muestra su gran versatilidad y cómo adapta el diseño al encargo y al promotor; la Plaza de Toros, el chalet de Fontecha, el Colegio Notarial o el Casino Primitivo son claros ejemplos de obras que han llegado hasta nuestros días. Los garajes, una tipología a medio camino entre la residencial y la industrial, reafirma la capacidad del arquitecto para adaptar sus diseños hacia el tipo de negocio al que van encaminados. Como ejemplo de ello, se puede observar el diseño del garaje-hospedería Moreno donde prima la funcionalidad de hospedería y taller sobre la de garaje, o los garajes Ibáñez y López Belmonte dónde los diseños de sus fachadas potencian más las actividades complementarias de exposición de vehículos y venta de accesorios que la propia función de garaje.

D. Francisco Fernández Molina fue arquitecto municipal desde el año 1927 hasta su muerte en el año 1929. Presenta cuatro obras en el período de estudio, tres de ellas correspondientes a particulares, probablemente clientes habituales suyos y otra el garaje Díaz, las cuáles son resueltas de una manera sobria y sin aspectos reseñables desde el punto de vista arquitectónico.

De D. Buenaventura Ferrando Castells, arquitecto valenciano y principal representante de la tendencia mediterránea modernista, se identifican solo dos obras en el período, una obra menor de garaje individual para D. José María Alcaraz y el edificio de viviendas para alquilar de D. Ángel González, en dónde aprovecha la planta baja para plantear un garaje. Se trata de un diseño sobrio, sin grandes alardes y sin recurrir al ornato que muestra en obras de la capital albaceteña como el Pasaje de Lodares o el edificio de la calle San Julián.

El último de los arquitectos es D. Miguel Ortiz e Iribas, autor de los garajes de D. Francisco López y de D. Gregorio Díaz. Se trata de dos obras menores, en las que la funcionalidad prima por encima de cualquier otro aspecto, y no reflejan el esplendor de obras anteriores y futuras de este arquitecto en la ciudad de Albacete como las Casas Cabot y el edificio del Archivo Histórico Provincial.

\section{Conclusiones}

El tamaño de la ciudad de Albacete influye en la aparición y en las características de los garajes. La mayoría de los garajes son individuales y pequeños, destinados principalmente a guarda y custodia de los vehículos. Las dos únicas instalaciones qué por sus características funcionales, de negocio y arquitectónicas, se podrían incluir dentro de la tipología de garaje que se identifica en las grandes ciudades son los garajes López Belmonte e Ibáñez. Estos garajes fueron diseñados en 1924 y 1925 respectivamente, más de una década después de la aparición de los garajes en Valencia y Buenos Aires, muestra inequívoca de la introducción y proliferación más tardía del automóvil en Albacete.

Los primeros garajes se articulan en dos zonas: el centro de la ciudad (facilitando el acceso a las instituciones y negocios) y en torno las carreteras de Ocaña a Alicante y la carretera de Jaén (reafirmando el concepto de posada y fonda que tiene la ubicación geográfica de la ciudad).

Los garajes se diseñan como edificaciones exprofeso, principalmente en planta baja, albergando alguna de las instalaciones una planta primera para los trabajadores o como posada de chóferes. Su arquitectura, a excepción de los diseños modernistas de Julio Carrilero de los garajes López Belmonte e Ibáñez, no presenta grandes alardes ni mucho ornato, se adapta el edificio al solar y a su uso, la guarda de los vehículos. 


\section{Agradecimientos}

Al personal del Archivo Municipal de Albacete por el apoyo y asistencia durante el período de búsqueda de los expedientes de obras y a los revisores que han evaluado el presente trabajo en aras de su perfeccionamiento.

Conflicto de intereses: El autor declara que no hay conflicto de intereses.

\section{Bibliografía}

Aguilar Civera, I. (2003). El territorio como proyecto. Transporte, obras públicas y ordenación territorial en la historia de la Comunidad Valenciana. Valencia, España: Generalitat Valenciana.

Archivo Municipal de Albacete (AMA). Expedientes de Obras. Albacete.

Archivo Histórico Provincial de Albacete (AHPA). Hemeroteca. Albacete.

Benito Goerlich, D. (1992). La arquitectura del eclecticismo en Valencia: vertientes de la arquitectura valenciana entre 1875 y 1925. Valencia, España: Ayuntamiento de Valencia.

Dirección General de Tráfico (DGT). (1965). Primeros vehículos matriculados en España (año 1965). Recuperado de http://www.dgt.es/images/Primeros-Vehiculos-matriculados-en-Espana-1900-1964Biblioteca-DGT-1008562.pdf

Elinbaum, P. (2018). La construcción social del planeamiento urbano. Elementos para la investigación empírica y la reflexión desde la práctica. ACE, Architecture, City and Environment, 12(36): 15-38. DOI: http://dx.doi.org/10.5821/ace.12.36.4729

Fuentes-Bargues, J.L. (2014). La arquitectura de los primeros garajes de Valencia. Scripta Nova. Revista Electrónica de Geografía y Ciencias Sociales, XVIII(466). Recuperado de http://www.ub.edu/geocrit/sn/sn-466.htm

Gutiérrez-Mozo, E. (2001). El despertar de una ciudad: Albacete 1898-1936. Madrid, España: Celeste Ediciones.

Gutiérrez-Mozo, E. (2004). Paseos de Arquitectura por la ciudad de Albacete. De la ilustración a la Modernidad. Albacete, España: La Siesta del Lobo.

Gutiérrez-Mozo, E. (2012). Albacete plural: Perspectiva de género y gestión de la participación en un plan de ordenación municipal. Arquitectura y Urbanismo, 33(1). Recuperado de http://scielo.sld.cu/scielo.php?script=sci arttext\&pid=S1815-58982012000100002

Gutiérrez-Mozo, D., Parra-Martínez, J., Martínez-Medina, A. (2020). La universidad laboral de Albacete (1974-75), un episodio olvidado en el legado arquitectónico de Julio Cano Lasso. ACE, Architecture, City and Environment, 15(43), 7316. DOI: http://dx.doi.org/10.5821/ace.15.43.7316

Instituto Nacional de Estadística (INE). (1955). Reseña Estadística de la provincia de Albacete. Madrid, España: María Gómez. 
Mac Donald, S. S. (2007). The parking garage: design and evolution of a modern urban form. Washington, USA: Urban Land Institute.

Magán Perales, J.M. (1997). El desarrollo urbanístico de la ciudad de Albacete. Albacete, España: Instituto de Estudios Albacetenses.

Martínez-Verón, J. (2017). Zaragoza Arquitectura del Siglo XX / Industrial /Garaje de Automóviles Lacarte. Recuperado de https://zaragozaarquitectura sigloxx.com/category/industrial/page/2/

Ministerio de Educación, Cultura y Deporte. (2015). Plan Nacional del Patrimonio Industrial. Recuperado de: https://sede.educacion.gob.es/publiventa/descarga.action?f codigo agc=15114C

Muñoz Carrión, A. (2012). El patrimonio cultural industrial: un lugar de encuentro. ArDIN: Arte, Diseño e Ingeniería, vol. 1, 43-53. Recuperado de http://polired.upm.es/index.php/ardin/article/view/1846

Muñoz, A. (2018). Espacios para la inmovilidad. La incidencia del automóvil en la arquitectura comercial de la ciudad de Buenos Aires entre 1887 y 1930. Seminario de Crítica, no 220. Recuperado de http://www.iaa.fadu.uba.ar/publicaciones/critica/0220.pdf

Panadero-Moya, M. (1984). La formación del "núcleo histórico" en la ciudad de Albacete. Boletín de Información "Cultural Albacete", 6. Recuperado de http://www.dipualba.es/archivo/estudios/la formaci\%C3\%B3n del n\%C3\%BAcleo hist\%C3\%B3rico .htm

Romero Soriano, R. M., Talavera Picazo, C. B., Romero Gombau, J. J. (1980). Notas para el estudio de la arquitectura de Albacete (1920-1930). Al-Basit: Revista de estudios albacetenses, vol. 8, 33-54. 